\title{
Multipath Exploitation in Non-LOS Urban Synthetic Aperture Radar
}

\author{
Pawan Setlur, Member, Tadahiro Negishi, Student Member, Natasha Devroye, Member, Danilo Erricolo, Senior \\ Member, IEEE
}

\begin{abstract}
Multipath is exploited to image targets that are hidden due to lack of line of sight (LOS) path in urban environments. Urban radar scenes include building walls, therefore creating reflections causing multipath returns. Conventional processing via synthetic aperture beamforming algorithms do not detect or localize the target at its true position. To remove these limitations, two multipath exploitation techniques to image a hidden target at its true location are presented under the assumptions that the locations of the reflecting walls are known and that the target multipath is resolvable and detectable. The first technique directly operates on the radar returns, whereas the second operates on the traditional beamformed image. Both these techniques mitigate the false alarms arising from the multipath while simultaneously permitting the shadowed target to be detected at its true location. While these techniques are general, they are examined for two important urban radar applications: detecting shadowed targets in an urban canyon, and detecting shadowed targets around corners.
\end{abstract}

Index Terms-Urban sensing, multipath, Ray-tracing, synthetic aperture radar, beamforming, point spread function

\section{INTRODUCTION}

$\mathbf{W}$ E propose two techniques to image a target inside urban environments, which are rich in multipath due to many sources of reflections, such as building walls. A novelty of the presented techniques is that they exploit multipath and they operate in the absence of LOS with the target. Conventional approaches do not consider multipath and would produce high false positives. Our approach takes advantage of the multipath false positives to associate and map them back to their true shadowed target location, thus reducing the false positives while increasing the signal to clutter ratio (SCR) at the shadowed target location.

The techniques are based on a ray tracing approach: One technique operates directly on the radar returns and its performance is dictated by a composite point spread function (CPSF), while the other technique operates on the beamformed image and utilizes the point spread function (PSF) explicitly to obtain the final image. Both techniques result in an image localizing and detecting the target at its true shadowed location, but vary in computational complexity, and requirements on the coherency of multipath.

Two examples of urban geometries are examined. The first is an urban canyon comprised of three building walls emulating an alley in a city but with an obstruction which

This work was performed when P. Setlur was at the University of Illinois at Chicago. $\mathrm{He}$ is now affiliated to Wright State Research Inst., as a research contractor with the US AFRL/RYAP, WPAFB, OH, email:pawan.setlur.ctr@wpafb.af.mil.

T. Negishi, N. Devroye, and D. Erricolo are with the Department of Electrical and Computer Engineering, University of Illinois at Chicago, Chicago, IL, 60607 USA, e-mail:t.negishi@gmail.com, $\{$ devroye,derric1\}@uic.edu. blocks the LOS with the target (a hidden target). The second is an urban T-junction where the target must be detected and localized around corners [1],[2]. In order to exploit multipath in urban terrain it is assumed that the blueprint of the urban scene is known to the radar a priori which may have been obtained for example through municipal blueprints or through military or civilian satellites or unmanned aerial vehicle (UAV) surveillance gathered previously. This is possible because, for example, the authors of [3] used information from the publicly available Google Earth to predict RF propagation in urban areas. Furthermore, radar systems may communicate with, or have other integrated sensors such as electro-optical (EO) /infrared (IR) sensors (for e.g. LIDAR) which provide high resolution and accurate ground elevation maps of city streets and urban canyons. While our algorithm assumes perfect knowledge of the reflecting geometry, we nonetheless show the effect of multipath exploitation when the errors exist in the blueprints through analytical and simulation results.

Assumptions. In this article it is assumed that: a) knowledge of the reflecting walls geometry is available; b) multipath can be treated as specular; c) all diffuse multipath can be treated as rough wall scattering; d) information on the type of walls for rough wall scattering is available; e) diffraction effects can be neglected; f) and, multipath returns are detectable and resolvable.

Literature on multipath exploitation. Multipath exploitation assuming perfect knowledge of the reflective geometry has been a recent subject of investigation, see for example [4]-[14]. Most articles have assumed a LOS path. In [4] the authors use knowledge of the environment to predict multipath and improve the probability of detection of the target. In [5], the authors use the multipath and the direct LOS path to track targets in an urban canyon with a particle filter and adaptive waveform design. In [6] the LOS paths were assumed present, in addition to the double bounce multipath. The radar returns were then jointly analyzed in the rangeDoppler domain but ghosts were not explicitly mapped or associated back to the target. An orthogonal frequency division multiplexing (OFDM) radar transmission scheme was used to exploit multipath in the presence of the LOS path in [7]. A generalized likelihood ratio test (GLRT) was then derived to detect targets using multipath and the direct LOS path, adaptive waveform design was considered in the formulation. Improvements in direction of arrival estimation using multipath exploitation were shown in [8]. Ground moving target indication (GMTI) applications were first treated in [9], in which Cramér-Rao bounds demonstrated that range accuracy may improve by exploiting NLOS multipath. 
In [10], [11], the authors project multipath echos into several auxiliary delay-image planes, which is claimed to result in better estimates of scattering centers via direct path image reconstruction. Synthetic aperture radar images demonstrating multipath ghosts due to a human target in a rectangular room were shown, e.g., in [12] via finite difference time domain (FDTD) electromagnetic modeling. The presence of the LOS path was assumed and the application was through-the-wall sensing.

In [13], the authors modify their SAR imaging algorithm to view shadowed regions of the target in the presence of LOS. Although we do the same to combat the absence of the LOS, our objective is different from [13] and focuses on associating and mapping the aspects viewed by the multipath back to the target's shadowed location. In [14], exploitation of single bounce multipath (ghost) targets in the presence of the target LOS paths was analyzed in the SAR domain for through-the-wall sensing. The objective here unlike [5]-[12] is to reduce false positives and improve the SCR at the target location by association and mapping of the multipath [14], [15].

Contributions. Our contributions may be summarized as follow: First, multipath exploitation in the SAR domain, and in the absence of the LOS path is addressed. Second, two techniques are derived to combat the absence of the LOS path in order to both localize and detect the target at its shadowed location. Third, the two techniques are applied to practical and commonly encountered urban scenarios, namely, shadowed urban canyons, where a target is hiding to avoid detection via radar, and detecting targets behind corners in urban city streets, such as an urban T-junction. Fourth, both image domain CFAR and rough wall simulations for multipath exploitation in the SAR domain have been addressed by numerical simulations.

Outline. In section II, brief preliminaries of traditional processing of free space radar returns with multiple sensors using a simple SAR backprojection technique is outlined. In section III, the example urban models, i.e., the urban canyon and the T-junction are considered and the multipath are analyzed. The techniques which exploit multipath to detect and localize the shadowed target are presented in section IV. Simulations are presented in section V, and we conclude in section V.

\section{Free space Imaging Preliminaries}

Assuming a free space scenario, consider a single point target at coordinates $\mathbf{x}_{t}=\left[x_{t}, y_{t}\right]^{T} \in \mathbb{R}^{2}$ and $M$ sensor positions $\mathbf{r}_{m} \in \mathbb{R}^{2}, m \in\{1,2, \cdots M\}$. The sensor at position $m$ transmits the waveform $s_{m}(t)$ and collects the radar returns, and then moves to the next sensor position, synthesizing a physical aperture. The waveform $s_{m}(t)$ for $t \in\left[(m-1)\left(T_{p}+T\right),(m-1)\left(T_{p}+T\right)+T\right]$ is defined as

$$
s_{m}(t)=p\left(t-(m-1) T_{p}\right) \exp \left(j \omega_{c}\left(t-(m-1) T_{p}\right)\right),
$$

where $t$ indexes the time, $\omega_{c}$ is the carrier frequency, $T$ is the waveform duration, and $T_{p}$ is time between two consecutive waveform transmissions at consecutive sensor positions. The waveform $p(t)$ is the baseband equivalent of the transmitted waveform and is assumed to be real. The received radar return at the $m$-th sensor position is given by $r_{m}(t)$,

$$
r_{m}(t)=\rho_{m} s_{m}\left(t-2 \tau\left(\mathbf{x}_{t} ; \mathbf{r}_{m}\right)\right)+v_{m}(t)
$$

where, $\tau(\mathbf{a} ; \mathbf{b})=\|\mathbf{a}-\mathbf{b}\| / c$ is a time-delay, $c$ is the speed of light in free space, $v_{m}(t)$ is noise plus interference observed by the radar at the $m$-th sensor position. Here, $\rho_{m}$ depends on the radar cross section (RCS) of the target and the attenuation due to the propagation of the signal observed by the radar at the m-th sensor position. Let $\mathcal{X}$ and $\mathcal{Y}$ be the discrete sets of $N_{x}$-crossrange and $N_{y}$-downrange positions, respectively. Consider an arbitrary position $\mathrm{x} \in \mathbb{R}^{2}$, the backprojected beamformed image pixel value at location $\mathrm{x}$, denoted with $\mathcal{I}(\mathbf{x})$, is given by [18]-[20],

$$
\mathcal{I}_{o}(\mathbf{x})=\left.\sum_{m=1}^{M}\left\{r_{m}\left(t+2 \tau\left(\mathbf{x} ; \mathbf{r}_{m}\right)\right) \circledast s_{m}^{*}(-t)\right\}\right|_{t=0}
$$

where $\circledast$ is the convolution operator. In practice, the timedelays $\tau(\cdot, \cdot)$ in (3) can be implemented off-line using the FFT via the Fourier (time) shift theorem. Repeating (3) for all the locations yields the beamformed image, $\mathcal{I}_{o}(\cdot) \in \mathbb{C}^{N_{x} \times N_{y}}$.

\section{MODEL}

To analyze our multipath exploitation algorithms, we consider two geometries modeling simplified city streets: an urban canyon and an urban T-junction.

\section{A. Urban Canyon}

Fig. 1 may model a practical scenario, such as a surveillance vehicle moving on a street but looking sideways into an alley for potential threats. Other practical scenarios may be envisioned for through-wall radar applications by inclusion of a front wall in the urban canyon. The length and width of the canyon are denoted by $D_{1}$ and $D_{2}$, respectively. Assume that the origin is at the intersection of the horizontal line through the $\mathrm{m}$-th sensor and the extension of wall 1 , denoted by $\mathrm{O}$ in Fig. 1, define the sensor locations $\mathbf{r}_{m}:=\left[-d_{m}, 0\right]^{T}$, and the target location $\mathbf{x}_{t}:=\left[-x_{t}, y_{t}\right]^{T}$. The LOS path from the target to the radar is absent because of an obstruction. However, the multipath from each wall to the target are present in the radar returns. Assuming specular reflections, each of these multipath returns create virtual targets at locations, $\mathbf{x}_{k}^{v t}, k=1,2,3$ corresponding to the three walls, and given by

$$
\begin{aligned}
& \mathbf{x}_{1}^{v t}:=\left[x_{t}, y_{t}\right]^{T}, \\
& \left.\mathbf{x}_{2}^{v t}:=\left[-x_{t}, 2\left(D_{1}+D_{y}\right)-y_{t}\right)\right]^{T}, \\
& \mathbf{x}_{3}^{v t}:=\left[-\left(2 D_{2}-x_{t}\right), y_{t}\right]^{T} .
\end{aligned}
$$

For ease of exposition, we consider models in 2D. However, 3D models could be handled by concatenating the radar and target heights to the vectors $\mathbf{r}_{m}, \mathbf{x}_{t}$, and the virtual targets heights to $\mathbf{x}_{k}^{v t}, k=1,2,3$, respectively. The imaging algorithm described in Section-II and the multipath exploitation algorithms would be unchanged except for incorporating the heights into the position vectors.

Since the LOS is absent, the radar returns at the $m-t h$ sensor is the superposition of pure multipath contributions, i.e.

$$
r_{m}(t)=\sum_{k=1}^{3} \rho_{m k} s_{m}\left(t-2 \tau\left(\mathbf{x}_{k}^{v t} ; \mathbf{r}_{m}\right)\right)+v_{m}(t) .
$$




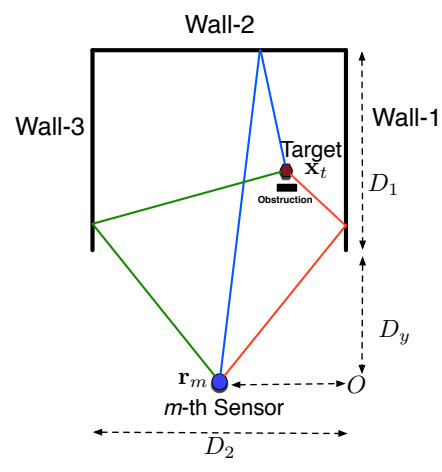

Fig. 1: Urban canyon

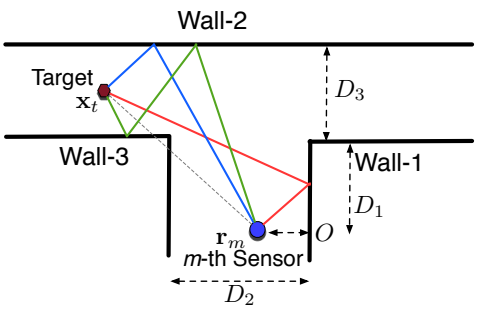

Fig. 2: T-junction
The three trajectories $k=1,2,3$ are different for each sensor position $m$ and, therefore, they are identified with the pair $(m, k)$. The coefficient $\rho_{m k}$ depends on the RCS of the target as it is seen by the $m$-th sensor, and upon the propagation attenuation along the $k$-th path.

Traditional processing of the radar returns as in (3) would indicate three targets due to the three multipath and the true target at $\mathbf{x}_{t}$ would go undetected.

Note that for ease of exposition, in (5), we have ignored radar returns that propagate from the radar to the target via one particular path but arrive back at the radar via a different path. Such combination multipath, if they exist, and are detectable, can be handled with straightforward extensions (though notationally more involved) of the multipath techniques presented subsequently. We briefly discuss this in the Simulations, Section VB.

Although the model in (5) implicitly considers the signal attenuation as a function of the distance, we have ignored its impact on the strength of the multipath in the simulations because our techniques do not depend on the relative strength of the multipath returns. We therefore note that, in practice, the signal attenuation or path loss phenomenon may preclude some or all of the multipath to be detected.

\section{B. Detection of targets around corners: Urban T-junction}

Consider Fig. 2, which depicts an urban T-junction. The origin is chosen as the point of intersection between the horizontal line through the sensor location and the vertical portion of wall 1 , denoted as $\mathrm{O}$. The coordinates $\mathbf{r}_{m}$ and $\mathbf{x}_{t}$ are identical to those defined for the urban canyon. The target is shadowed by wall 3 . Hence the direct LOS path is absent. However, there exist three multipath returns: two from reflections at wall 1,2 respectively, the third from a reflection at wall 3 and another at wall 2. Again assuming specular reflection, each of these multipath returns will give rise to three virtual targets, denoted as $\mathbf{y}_{k}^{v t}, k=1,2,3$, at

$$
\begin{aligned}
& \mathbf{y}_{1}^{v t}=\left[x_{t}, y_{t}\right]^{T}, \\
& \mathbf{y}_{2}^{v t}=\left[-x_{t}, 2\left(D_{3}+D_{1}\right)-y_{t}\right]^{T}, \\
& \mathbf{y}_{3}^{v t}=\left[-x_{t}, 2 D_{3}+, y_{t}\right]^{T} .
\end{aligned}
$$

The radar return at the $m$-th sensor position is identical to (5) after replacing $\mathbf{x}_{k}^{v t}$ with $\mathbf{y}_{k}^{v t}$. Traditional processing of the radar returns as in (3) will indicate three targets due to the three multipaths and the true target at $\mathbf{x}_{t}$ goes undetected.

For the LOS path to be absent for all sensor positions in this scenario, the $\left(x_{t}, y_{t}\right)$ must be in the following region (set):

$$
\bigcap_{m=1}^{M}\left\{\left(x_{t}, y_{t}\right): \frac{y_{t}\left(d_{m}-D_{2}\right)}{d_{m}-x_{t}} \leq D_{1}\right\}
$$

In the same spirit, we have until now assumed that all of the depicted multipath returns are present in the radar returns. This is in general not true, and the target co-ordinates must be in a region for all of the multipath to be physically present in the radar returns. For example, for the multipath from wall 1 to be present in all the $m$ radar range profiles implies that the target co-ordinates must be in the set:

$$
\bigcap_{m=1}^{M}\left\{\left(x_{t}, y_{t}\right): \frac{y_{t} d_{m}}{\left(x_{t}+d_{m}\right)} \leq D_{1}\right\} .
$$

Likewise, for multipath comprising of reflection at wall 2 to be present in $m$ profiles implies the following region:

$$
\bigcap_{m=1}^{M}\left\{\left(x_{t}, y_{t}\right): \frac{\left(d_{m}-D_{2}\right)\left(2\left(D_{3}+D_{1}\right)-y_{t}\right)}{d_{m}-x_{t}} \geq D_{1}\right\}
$$

Similarly, for the multipath comprising of reflections at wall 3 and wall 2 to be observed in all the $m$ range profiles, the following may be derived:

$$
\begin{aligned}
& \bigcap_{m=1}^{M}\left\{\left(x_{t}, y_{t}\right): \frac{\left(d_{m}-D_{2}\right)\left(2 D_{3}+y_{t}\right)}{d_{m}-x_{t}} \geq D_{1}\right\} \\
& \bigcap_{m=1}^{M}\left\{\left(x_{t}, y_{t}\right): \frac{x_{o}\left(x_{t}, y_{t}\right)\left(y_{t}-D_{1}\right)+x_{t}\left(D_{1}-y_{o}\left(x_{t}, y_{t}\right)\right)}{y_{o}\left(x_{t}, y_{t}\right)+y_{t}-2 D_{1}} \leq-D_{2}\right\} \\
& x_{o}\left(x_{t}, y_{t}\right):=\frac{D_{1}\left(d_{m}-x_{t}\right)-D_{3}\left(d_{m}+x_{t}\right)-y_{t} d_{m}}{2 D_{3}+y_{t}} \\
& y_{o}\left(x_{t}, y_{t}\right):=D_{3}+D_{1}
\end{aligned}
$$

In (8)-(10) the sets represent target positions where multipath is present for all the sensor positions. Furthermore, in (9),(10) the sets considered multipath from the left side of the Tjunction, similar counterparts may be derived for the right half in a straightforward manner. In reality, some sensors may receive a particular multipath while others do not; scenarios when the spatial strength of some multipath are below the 
noise floor are treated in the simulations. The LOS path is assumed to always be absent. The sets similar to (7)-(10) may be derived from the geometry of the urban canyon as in Fig. 1, and are not discussed here, as they are straightforward.

So far diffraction effects have been ignored as they are very particular to the geometry and are not always present. A ray incident on a diffracting edge causes a whole cone of diffracted rays and one needs to select how many trajectories should be tracked from this cone. Overall this process may easily become quite complicated. In many cases, rays originating from diffraction are weaker than rays originating from reflections. Therefore, after a few of such interactions it is usually correct to eliminate diffracted contributions because they are weaker overall compared to those originating solely from reflections. Note however, if one can model the diffraction a priori, for example via EM simulations, it may be possible to exploit some of it, i.e. map and associate the weaker diffraction ghosts back to the target, if they are detectable in the first place.

\section{Multipath Exploitation}

Our objective is to mitigate false positives and localize/detect the shadowed target through knowledge of the scene geometry. In this section, the multipath exploitation algorithms are presented in full generality. Let us denote the virtual targets as $\chi_{k}^{v t}, k=1,2,3$ for the urban canyon or the $\mathrm{T}$ junction Specifically, for the urban canyon $\chi_{k}^{v t}=\mathbf{x}_{k}^{v t}$ and for the T-junction $\chi_{k}^{v t}=\mathbf{y}_{k}^{v t}$. Let $\mathcal{W}=\mathcal{X} \times \mathcal{Y}$ be the set of the crossrange and downrange positions both inside and outside the canyon, and the T-junction, and let $\mathcal{W}_{\text {sub }}=$ $\left(\mathcal{X}_{\text {sub }} \times \mathcal{Y}_{\text {sub }}\right) \subseteq \mathcal{W}$ denote the subset of positions consisting of only those crossrange and downrange positions inside the urban canyon and T-junction, where $\mathcal{X}_{\text {sub }} \subseteq \mathcal{X}$ and $\mathcal{Y}_{\text {sub }} \subseteq \mathcal{Y}$ are of cardinality $N_{x 1}$ and $N_{y 1}$, respectively.

\section{A. Data Domain}

Define an arbitrary location $\mathbf{x}:=[-x, y]^{T} \in \mathcal{W}_{\text {sub }}$ with three associated multipath virtual targets $\bar{\chi}_{k}^{v t}, k=1,2,3$, as in (4) and (6), but with $x_{t}$ replaced by $x$ and $y_{t}$ replaced by $y$. The multipath exploitation algorithm is: consider a modified beamformed image whose value at $\mathrm{x}$ is given by

$$
\begin{aligned}
\mathcal{I}_{1}(\mathbf{x}) & =\left.\sum_{m=1}^{M} \sum_{k=1}^{3}\left\{r_{m}\left(t+2 \tau\left(\bar{\chi}_{k}^{v t} ; \mathbf{r}_{m}\right)\right) \circledast s_{m}^{*}(-t)\right\}\right|_{t=0} \\
\text { for } r_{m}(t) & =\sum_{k=1}^{3} \rho_{m k} s_{m}\left(t-2 \tau\left(\boldsymbol{\chi}_{k}^{v t} ; \mathbf{r}_{m}\right)\right)+v_{m}(t)
\end{aligned}
$$

Equation (11) is linear in all operations and is a modified version of the original beamforming algorithm in (3), with a few important differences. The modified beamforming algorithm derived in (11) coherently adds $k=1,2,3$ multipath and associates them to the location $\mathbf{x}$. In contrast, the original imaging algorithm in (3) coherently adds the LOS return only (assuming the target were at $\mathbf{x}$ ), which in our case does not exist. We have therefore mitigated the false positives in the original beamformed image and rendered the target at its true location, which is clearly unattainable with the processing in (3) due to the absence of the target LOS path. The algorithm is presented in Table. I.
1) Composite Point Spread Function: To analyze the effects of (11) in the image domain, and see how it relates to the traditional point spread function (PSF) [17],[21], we make certain assumptions. Consider deriving the traditional PSF from (3) in the absence of noise and interference, i.e. $v_{m}(t)=0$, assuming zero path loss and under unit reflectivity, i.e. $\rho_{m}=1, \forall m$ (by definition of the PSF). To derive a function akin to a PSF but from (11), identical assumptions are therefore made, albeit on the multipath instead. That is, let $v_{m}(t)=0$, multipath be point like, have no path loss and have unit reflectivities, i.e. $\rho_{m k}=1$, for all $k=1,2,3, m=1,2, \ldots, M$. We define this PSF like function as a composite PSF (CPSF) for reasons explained shortly. If we denote the CPSF as $\mathcal{P}_{c}\left(\mathbf{x}_{t}, \mathbf{x}\right)$, then using elementary Fourier properties it may be shown that

$$
\begin{aligned}
& \mathcal{P}_{c}\left(\mathbf{x}_{t}, \mathbf{x}\right)=\sum_{m=1}^{M} \sum_{k=1}^{3} \sum_{l=1}^{3} \mathcal{F}^{-1}\left\{\left|P\left(\omega-\omega_{c}\right)\right|^{2}\right. \\
& \times \exp \left(-j\left(\omega-\omega_{c}\right) 2 \Delta \tau_{m k l}\left(\boldsymbol{\chi}_{k}^{v t} ; \overline{\boldsymbol{\chi}}_{l}^{v t}\right)\right) \\
& \left.\times \exp \left(-j \omega_{c} 2 \Delta \tau_{m k l}\left(\boldsymbol{\chi}_{k}^{v t} ; \overline{\boldsymbol{\chi}}_{l}^{v t}\right)\right)\right\}\left.\right|_{t=0}
\end{aligned}
$$

where $\Delta \tau_{m k l}\left(\boldsymbol{\chi}_{k}^{v t} ; \overline{\boldsymbol{\chi}}_{l}^{v t}\right):=\tau\left(\boldsymbol{\chi}_{k}^{v t} ; \mathbf{r}_{m}\right)-\tau\left(\overline{\boldsymbol{\chi}}_{l}^{v t} ; \mathbf{r}_{m}\right)$. The CPSF could be broken down into two additive terms: the desired term and a term causing spatial interference.

The desired term in the CPSF is given by

$$
\begin{aligned}
& \mathcal{Q}_{c}\left(\mathbf{x}_{t}, \mathbf{x}\right)=\sum_{m=1}^{M} \sum_{k=1}^{3} \mathcal{F}^{-1}\left\{\left|P\left(\omega-\omega_{c}\right)\right|^{2}\right. \\
& \times \exp \left(-j\left(\omega-\omega_{c}\right) 2 \Delta \tau_{m k k}\left(\boldsymbol{\chi}_{k}^{v t} ; \overline{\boldsymbol{\chi}}_{k}^{v t}\right)\right) \\
& \left.\times \exp \left(-j \omega_{c} 2 \Delta \tau_{m k k}\left(\boldsymbol{\chi}_{k}^{v t} ; \overline{\boldsymbol{\chi}}_{k}^{v t}\right)\right)\right\}\left.\right|_{t=0}
\end{aligned}
$$

It is noted that (13) is obtained from (12) when $k=l$. The interference term in (12) is given by $\mathcal{P}_{c}\left(\mathbf{x}_{t}, \mathbf{x}\right)-\mathcal{Q}_{c}\left(\mathbf{x}_{t}, \mathbf{x}\right)$.

When the LOS path for the target exists, the traditional PSF at an arbitrary location $\mathbf{x}$ evaluated using (3) is

$$
\begin{aligned}
& \mathcal{P}\left(\mathbf{x}_{t}, \mathbf{x}\right)=\sum_{m=1}^{M} \mathcal{F}^{-1}\left\{\left|P\left(\omega-\omega_{c}\right)\right|^{2}\right. \\
& \left.\exp \left(-j\left(\omega-\omega_{c}\right) 2 \Delta \tau_{m}\left(\mathbf{x}_{t} ; \mathbf{x}\right)\right) \exp \left(-j \omega_{c} 2 \Delta \tau_{m}\left(\mathbf{x}_{t} ; \mathbf{x}\right)\right)\right\}\left.\right|_{t=0} \\
& \Delta \tau_{m}\left(\mathbf{x}_{t} ; \mathbf{x}\right):=\tau\left(\mathbf{x}_{t} ; \mathbf{r}_{m}\right)-\tau\left(\mathbf{x} ; \mathbf{r}_{m}\right)
\end{aligned}
$$

We may treat the multipaths as targets, and their PSF's may also be evaluated from (14) by substituting $\mathbf{x}_{t}$ with any one of the multipath virtual targets whose locations are given in (4) and (6). This is employed in the multipath exploitation algorithm in the image domain, treated subsequently. Comparing (13) and (14), we see that the CPSF is identical in structure to the PSF, and has several terms resembling the PSF, albeit considering the true multipath and its hypothesized multipath in (13) rather than the true target and a hypothesized target as in (14). This is no surprise given the similarity of (3) and (11).

It is tempting to define the resulting CPSF a 'PSF' for the modified beamforming algorithm in (11), but we refrain from doing so as the former has an interference term, while the latter has only sidelobes and possesses no interference terms. More importantly, the PSF by definition is evaluated for a single point target return [21], whereas the CPSF operates on the returns from several assumed point like multipath targets. 
TABLE I: Algorithm for multipath exploitation in data domain

1. Position select: Select an arbitrary position $\mathrm{x} \in \mathcal{W}_{\text {sub }}$ for the geometry selected

2. Initialize: Set $m=1$ and $\theta_{M}=0$. Using (4) or (6) compute the locations of the multipath for the arbitrary position $\mathbf{x}$ as well as its corresponding multipath time delays, $\tau\left(\overline{\boldsymbol{\chi}}_{k}^{v t} ; \mathbf{r}_{m}\right), k=1,2,3$.

3. Fourier operation: For the received radar return at the $m$-th sensor, $r_{m}(t)$, obtain $r_{m}\left(t+\tau\left(\overline{\boldsymbol{\chi}}_{k}^{v t} ; \mathbf{r}_{m}\right)\right)$ by using the FFT first on $r_{m}(t)$, appropriately modulating it in phase, and then taking the inverse FFT.

4. Match filter \& Range register: Compute $\theta_{m k}=\left.r_{m}\left(t+\tau\left(\bar{\chi}_{k}^{v t} ; \mathbf{r}_{m}\right)\right) \circledast s_{m}^{*}(-t)\right|_{t=0}, k=1,2,3$ and store $\theta_{M}=\theta_{M}+\sum_{k=1}^{3} \theta_{m k}$

5. Repeat: Increment $m$, if $m=M+1$, go to step-6, else go back to step-3.

6. Associate: Store the $\operatorname{cost} \theta_{M}$ to the matrix $I_{1}(\mathbf{x})$.

7. Check: Goto step-8 if all $\mathbf{x} \in \mathcal{W}_{\text {sub }}$ are exhausted, or else go back to step-2 and repeat.

8. Stop: The multipath exploited beamformed image (matrix) $\mathcal{I}_{1}(\cdot)$ now displays the shadowed target.

Theoretically the PSF is a spatial transfer function defined at the origin convolved with a spatial delta function at the target coordinates [21],[25]. The convolution is already computed in (14). For the CPSF a similar analogy cannot be formulated.

Both the CPSF and PSF derived in (13) and (14) are derived in the RF domain, but they may be converted into their equivalent baseband versions by replacing $\omega-\omega_{c}$ by $\omega$. This is useful in simulating the CPSF and PSF in section IV. The PSF and CPSF are dependent on $p(t)$ or, intuitively, its content in the spectral domain. Numerical results will be presented in Section IV for a rectangular waveform, and the pertinent analysis is relegated to Appendix-A. It is shown that in certain cases the interference term is spatially spread in both downrange and crossrange, and its magnitude is more or less insignificant when compared to the sidelobe levels of the original PSF. The resulting PSF and CPSF may be re-derived for other waveforms in a straightforward manner.

\section{B. Image Domain}

The image domain algorithm is similar to the approach taken in [14] but has two major differences. First, it uses the PSF to perform weighting rather an arbitrary and suboptimal spherical weighting function as employed in [14]. The spherical weighting may cause nearby clutter and interference to be mapped back to the target location, which is avoided by using the PSF. Moreover radar SAR algorithms usually employ the PSF theory and was absent in [14]. Second, the algorithm is formulated to address the absence of LOS, which was never the focus of [14] that was aimed at through-wall radar applications.

Consider an arbitrary location $\mathbf{x} \in \mathcal{W}$ and a hypothesized target at location $\breve{\mathbf{x}}_{t} \in \mathcal{W}_{s u b}$. The hypothesized multipath are then located at $\breve{\chi}_{k}^{v t}, k=1,2,3$, as may be obtained from (4) and (6).

Let us evaluate the PSFs at $\breve{\chi}_{k}^{v t}$ in $\mathcal{W}$ and denote them in a matrix notation $\mathbf{P}_{k}\left(\breve{\chi}_{k}^{v t}\right), k=1,2,3$. In other words, $\mathcal{P}\left(\breve{\chi}_{k}^{v t} ; \mathbf{x}\right)$ is an element of $\mathbf{P}_{k}\left(\breve{\chi}_{k}^{v t}\right)$ indexed appropriately. For subsequent operations on the matrices $\mathbf{P}_{k}\left(\breve{\chi}_{k}^{v t}\right)$, we normalize them as

$$
\overline{\mathbf{P}}_{k}:=\frac{\left|\mathbf{P}_{k}\left(\breve{\chi}_{k}^{v t}\right)\right|}{\left\|\mathbf{P}_{k}\left(\breve{\chi}_{k}^{v t}\right)\right\|_{\max }}, k=1,2,3
$$

$$
\left\|\mathbf{P}_{k}\left(\breve{\chi}_{k}^{v t}\right)\right\|_{\max }=\max _{\mathbf{x}}\left\{\left|\mathcal{P}\left(\breve{\chi}_{k}^{v t} ; \mathbf{x}\right)\right|\right\}
$$

where, $|\cdot|$ denotes the absolute value that we allow to operate on matrices elementwise as well. A simple thresholding operation is now employed with threshold parameter $\beta$ given by,

$$
\breve{\mathbf{P}}_{k}=\mathbb{1}_{\left[\overline{\mathbf{P}}_{k} \leq \beta\right]}
$$

where the $\mathbb{1}_{[.<.]}$as the indicator function and we define it to operate on the matrix element-wise as well. The matrices $\breve{\mathbf{P}}_{k}, k=1,2,3$ are now binary matrices/images and typically consist of many 1 's in the vicinity of the hypothesized multipath location, $\breve{\chi}_{k}^{v t}$, depending on the choice of the threshold parameter $\beta$. We convert the normalized PSFs to a $\mathrm{dB}$ scale and choose $\beta$ such that only the main lobe of the hypothesized multipath at $\breve{\chi}_{k}^{v t}$ is selected for further processing. Typically the range of $\beta$ is between $3 \mathrm{~dB}-13 \mathrm{~dB}$.

The multipath exploitation technique now consists of a masking operation on the original beamformed image,

$$
\mathbf{I}_{\text {mask }}\left(\breve{\mathbf{x}}_{t}\right)=\left|\mathbf{I}_{o}\right| \odot\left(\breve{\mathbf{P}}_{1}+\breve{\mathbf{P}}_{2}+\breve{\mathbf{P}}_{3}\right)
$$

where $\odot$ is the Hadamard product or elementwise multiplication and $\mathbf{I}_{o}$ is the matrix equivalent of the original beamformed image $\mathcal{I}_{o}$. It is seen from (17) that if there were hypothesized multipath at $\breve{\chi}_{k}^{v t}$, associated with the shadowed target at $\breve{\mathbf{x}}_{t}$, then it would be retained in $\mathbf{I}_{\text {mask }}(\cdot)$, while nulling the clutter and interference in the beamformed image, whose matrix equivalent is denoted as $\mathbf{I}_{o}$. In contrast, if there were no multipath at the hypothesized multipath, associated with the shadowed target at $\breve{\mathbf{x}}_{t}$, then (17) is a null image consisting of all zeros. The final step is an association stage which maps the multipath energy to its hypothesized target location $\breve{\mathbf{x}}_{t}$.

$$
\mathcal{I}_{e}\left(\breve{\mathbf{x}}_{t}\right)=\mathbf{1}_{N_{x}}^{T} \mathbf{I}_{\text {mask }}\left(\breve{\mathbf{x}}_{t}\right) \mathbf{1}_{N_{y}}
$$

where $\mathbf{1}_{N_{x}}$ and $\mathbf{1}_{N_{y}}$ are vectors of all ones and of dimensions $N_{x} \times 1$ and $N_{y} \times 1$, respectively. Equations (15)-(18) are repeated for all hypothesized target locations in $\mathcal{W}_{\text {sub }}$. The multipath exploitation technique in the image domain is now complete and the algorithm details are presented in Table. II. To avoid repetition, certain details are however omitted such as, computing the beamformed image in step- 2 of Table. II, which is straightforward from (3) and Table. I. 
TABLE II: Algorithm for multipath exploitation in image domain

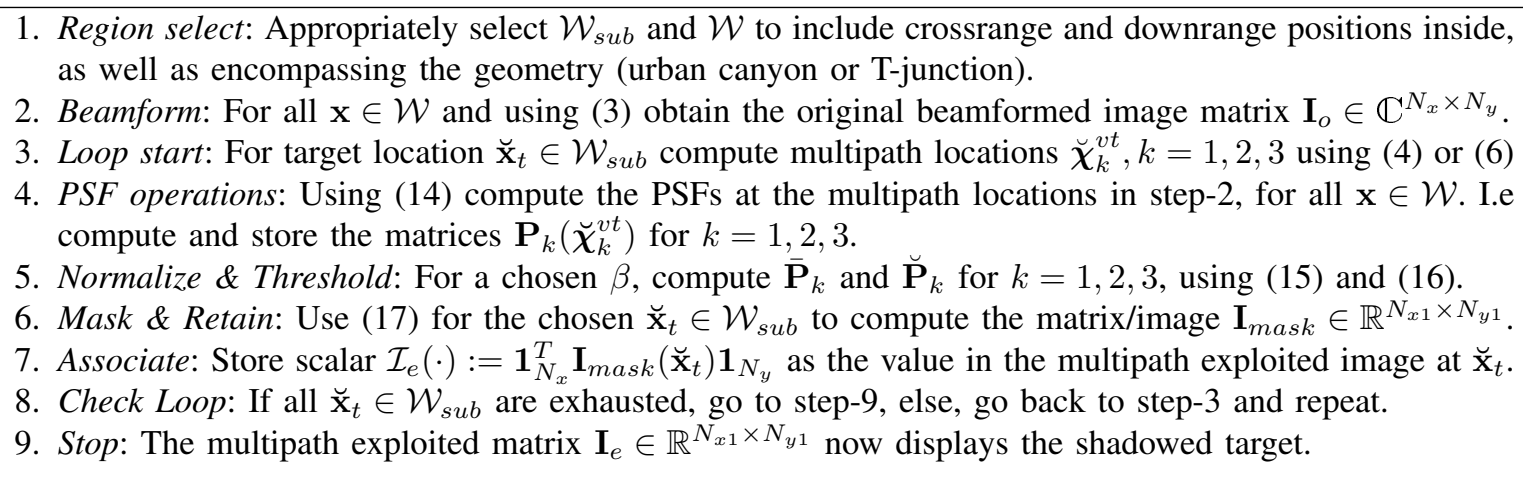

We now compare the two presented multipath exploitation techniques. The first approach computes the multipath exploited image depicting the target at its true shadowed location directly from the radar returns. The second approach operates on the classical beamformed image, which requires this to be computed beforehand. Thus, the first approach has computational advantages over the second. The second approach uses information from the beamformed image and hence spatial clutter or range profile artifacts could be traced from the beamformed image to the final multipath exploited image. On the other hand, the first approach directly maps the clutter and artifacts to the final exploited image, and thus poses difficulties in tracing them back to their original spatial origins in the absence of the original beamformed image. Other differences between the two techniques will be seen from the simulations in section $\mathrm{V}$.

So far, we have ignored clutter objects in the two techniques for multipath exploitation. We note that differentiating clutter, i.e. uninteresting targets from the true targets of interest is difficult, unless the clutter objects are stationary, in which case SAR MTI /GMTI and change detection techniques could be used. If clutter objects have their own multipath but no LOS paths, then our algorithm does not differentiate between the true and clutter targets.

\section{Multipath exploitation in non-ideal scenarios: rough walls}

Roughness should be taken into account when walls are not smooth, which typically occurs at shorter wavelengths. Without embarking into a rigorous electromagnetic analysis, which would require knowledge of material parameters that would not available in actual applications, we provide a simplified investigation to approximate the impact of wall roughness.

We use random perturbations to model roughness, as first pioneered by Rice [22], and used in for example [23][24] and references therein. To emulate roughness / craters on the wall, we consider $N$ subreflectors, which form the length of the wall(s) [6]. Each subreflector is placed at a random depth from the baseline smooth wall, according to a Gaussian distribution with mean value corresponding to the location of the baseline smooth walls and standard deviation corresponding to a percentage of the operating wavelength [6]. Pattern roughness or texture is simulated through spatial

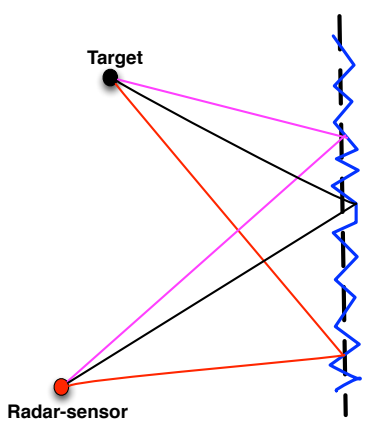

Fig. 3: Wall roughness modeling. Craters are shown exaggerated w.r.t. to the wavelength used. The baseline smooth wall (dashed black) and three diffuse multipath from three craters are shown.

correlation [6], [22] of the random subreflectors, so that high spatial correlation corresponds to presence of a pattern. The multipath returns from the random subreflectors are then weighted. We propose a simple but flexible weighting model based on several physical realities. Since it may be shown that the length of the specular multipath component is smaller than any of the diffuse multipath components, the weighting function is designed as follows: if a subreflector is closer to the specular point, the overall path length traveled is shorter (and hence experiences lower path loss) and its diffuse multipath component is weighted higher.

The following makes the modeling clearer. Referring to Fig. 3, consider a single rough vertical wall consisting of $N$ subreflectors. If the wall is smooth, then the multipath is specular and let us denote $\mathbf{x}_{s}:=\left[x_{s}, y_{s}\right]^{T}$ as the specular reflection point on the wall. The $n$-th subreflector has coordinates $\mathbf{x}_{\mathbf{n}}:=\left[x_{n r}, y_{n r}\right]^{T}$. Denote $\mathcal{N}(0,1)$ the standard normal distribution with zero mean and unit standard deviation and $\lambda$ the operating wavelength. Then $x_{n r}$ is random and modeled as $x_{n r} \sim \mathcal{N}\left(d_{x r}, \eta \lambda\right)$, with $\eta=$ wall roughness percentage. The baseline smooth wall is located $d_{x r}$ meters along the $x$ coordinate from an arbitrary origin. The $y$-coordinate of the $n$-th subreflector is deterministic with $y_{n r} \in\left[D_{y 1 r}, D_{y 2 r}\right]$, where $D_{y 2 r}-D_{y 1 r}$ is the vertical length of the wall. The time delay for the $n$-th diffuse multipath may then be obtained. The amplitude weighting associated with the diffuse multipath 
from this subreflector is given by $\mathcal{G}(n):=\exp \left(-\varepsilon\left|x_{s}-x_{n r}\right|\right)$, where $\varepsilon$ is a free parameter. The spatial correlation between two subreflectors is denoted as $\mathcal{S}\left(n_{1}, n_{2}\right):=\mathbb{E}\left\{\left(x_{n_{1} r}-\right.\right.$ $\left.\left.d_{x r}\right)\left(x_{n_{2} r}-d_{x r}\right)\right\},\left(n_{1}, n_{2}\right) \in(1,2, \ldots, N)$ and assumed to be $\mathcal{S}\left(n_{1}, n_{2}\right)=\exp \left(-\kappa\left|n_{1}-n_{2}\right|\right)$. The diffuse multipath from the $N$ subreflectors are now superposed. The technique to generate diffuse multipath from a single rough wall, parallel to the $y$ axis is summarized in Table III for a single sensor position and this technique could be extended to other geometries.

\section{Inaccuracies in wall positions}

When wall locations are not know with accuracy, our extensive simulations indicate two possible scenarios. In one, some multipath trajectories intersect and the simplest choice is to declare the intersection points as the localized target positions. In the other, none of the multipath trajectories intersect. Hence, we assume that the target is declared within a contiguous region in the SAR image to derive some confidence on the target location. In Appendix-B, we define an uncertainty polygon to represent partial confidence on the target's location.

\section{Simulations}

All dimensions are in meters, unless mentioned otherwise. The carrier frequency is $1.8 \mathrm{GHz}, 12$ sensors are used for imaging, with inter-element spacing equal to the wavelength divided by a factor of 4 to satisfy the spatial Nyquist. For the urban canyon, $D_{1}=D_{2}=20$, and the standoff distance is $D_{y}=4$. For the T-junction, $D_{1}=26, D_{2}=D_{3}=20$. The spatial sampling in both downrange and crossrange are kept constant in the simulations, however their total dimensions are varied to show the multipath which are outside the imaged geometries, and clearly indicated in the images themselves. When some parameters change, they are stated explicitly. The path loss or signal attenuation as a function of range has been ignored in the simulations.

\section{A. Ideal Scenarios:}

For the urban canyon, the target is at spatial coordinates ($12,14)$ and for the T-junction the target is at $(-22,30)$. The sensor start location is at $(-9.5417,0)$ and its end location is at $(-10,0)$. No noise or clutter was added in the radar returns and the targets were assumed to have unit reflectivity over all azimuths. Fig. 4 and Fig. 5 show results for both geometries and techniques. The walls comprising the geometry are superimposed on the figure and target locations are marked as ' $O$ '. Due to the absence of the direct path in Fig. 4(a), no target is observed inside the urban canyon geometry and the multipath are seen outside the urban canyon. For the T-junction in Fig. 5(a), one of the multipath is localized inside, and the rest of the multipath are localized outside. The multipath inside in T-junction in Fig. 5(a) is caused from the reflection at wall 1 , and not surprisingly is at the same downrange position as the target but appears on the opposite side of the genuine target. Fig. 5(a) falsely indicates that a target exists on the opposite side of the T-junction. The target is missed for imaging performed inside both geometries and multipath causes false alarms for imaging both inside and outside the geometries.
The data domain multipath exploitation images of Fig. 4(b) and Fig. 5(b) show that the multipath in Fig. 4(a) and Fig. 5(a) are "literally" folded back and intersect at the target location, in addition to a strong peak at the shadowed target location. Similar intersections of the corresponding multipath and the strong peak at the target location are seen for the image domain multipath exploited image in Fig. 4(c) and Fig. 5(c).

Considering the data domain exploitation algorithm in (11) the strong peak is designed, whereas the intersections are an added bonus. It is surprising, however, that the same intersections and the strong peak at the target location are seen for both geometries w.r.t. to the image domain algorithm though the intersections and the peaks appear to be "fatter" than those in the data domain algorithm. This could be attributed to the noncoherent spatial integration of the amplitudes at locations in the mainlobe of the multipath in the original beamformed images, and is explained by (18). Spatially spread interference due to the folding back of the multipath are seen for the data domain algorithm in Fig. 4(b) and Fig. 5(b). Similar trajectories are also seen in the image domain algorithm in Fig. 4(c) and Fig. 5(c). This implies that the data domain and image domain multipath exploitation algorithms are related but it is difficult to definitively point out this relationship from (11) and (15)-(18). From Fig. 4 and Fig. 5, we see that the data domain and image domain multipath exploitation has increased the SCR at the target location, and simultaneously reduced the false alarms. From here onward all images shown are normalized to their maxima, i.e. the gain in SCR at the target location is not shown explicitly.

\section{B. Combination Multipath}

In practice we could encounter situations where the propagation and return paths are different. Exploiting the combination multipath is straightforward and involves minor modifications to the techniques presented thus far. A simulation example for the data domain algorithm is shown in Fig. 6 for the urban canyon, where we considered a combination multipath propagating via wall 1 and returning via wall 2 . In total there are now four multipath including this additional combination multipath. We see that four multipath trajectories now intersect at the target location. For the image domain algorithm, the focus locations of the combination multipath are required, which are obtained from techniques used in [15]. After computing the focused locations of combination multipath, exploiting them is straightforward from the image domain exploitation technique presented here.

\section{Incoherent multipath}

Consider the unlikely scenario where the multipath add destructively across the sensor positions (the complex gains of the multiple paths were artificially chosen in this way). In Fig. 7, the data domain technique images are shown for the two previous geometries and the same target coordinates. From the inset in Fig. 7, we can see that the target location has a null in both the urban canyon as well as the T-junction. However, the target location may still be discernible from the structure of the images, i.e the folding and intersection of the multipath at the target location. Notice that the third multipath is stronger 
TABLE III: Modeling diffuse multipath radar returns from rough walls

1. Consider: A rough wall parallel to the $y$-axis of length $D_{y r}$, and at a distance of $d_{x r}$ from an arbitrary origin.

2. Compute: For the radar at coordinates given in $\mathbf{x}_{\mathbf{r}}$, target at $\mathbf{x}_{\mathbf{t}}$, and from geometry, compute the specular reflection coordinates and store them in the vector $\mathbf{x}_{\mathbf{s}}=\left[x_{s}, y_{s}\right]^{T}$.

3. Grid: Divide the length of the wall into $N$ subreflectors. The wall roughness percentage is $\eta$, operating wavelength, $\lambda$, and define $D_{y r}:=D_{y 2 r}-D_{y 1 r}$. The $n$-th subreflector on the rough wall is at $x-y$ coordinates $\left(x_{n r}, y_{n r}\right)$, where $x_{n r} \sim \mathcal{N}\left(d_{x r}, \eta \lambda\right)$ is random, and $y_{n r} \in\left[D_{y 1 r}, D_{y 2 r}\right]$ is deterministic. At this stage, the random components of the arbitrary, $n_{1}$-th and $n_{2}$-th subreflectors may be made correlated according to any valid correlation function, $\mathcal{S}\left(n_{1}, n_{2}\right)$.

4. Generate: For the $n$-th subreflector, generate a diffuse multipath component as $\mathcal{G}(n) s\left(t-2 \tau_{n}\right)$, where $\tau_{n}:=|| \mathbf{x}_{\mathbf{r}}-\mathbf{x}_{\mathbf{n}} \| / c+|| \mathbf{x}_{\mathbf{n}}-\mathbf{x}_{\mathbf{t}}|| / c, \mathbf{x}_{\mathbf{n}}:=\left[x_{n r}, y_{n r}\right]^{T}$, and for a predefined $\varepsilon, \mathcal{G}(n):=\exp \left(-\varepsilon\left|x_{s}-x_{n r}\right|\right)$.

5. Loop: Repeat the processing in step-4 for a different $n$, until all the $N$ subreflectors have been exhausted. The composite diffuse multipath returns is then given by $\sum_{n=1}^{N} \mathcal{G}(n) s\left(t-2 \tau_{n}\right)$.
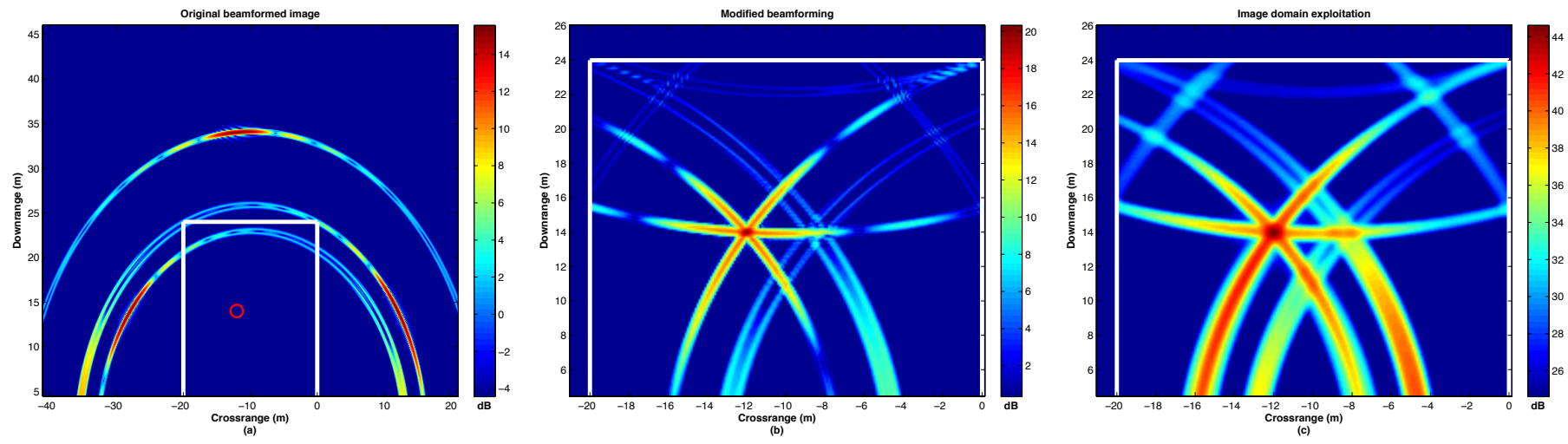

Fig. 4: For Urban canyon: (a) original beamformed, and multipath exploited (b) in data domain, (c) in image domain
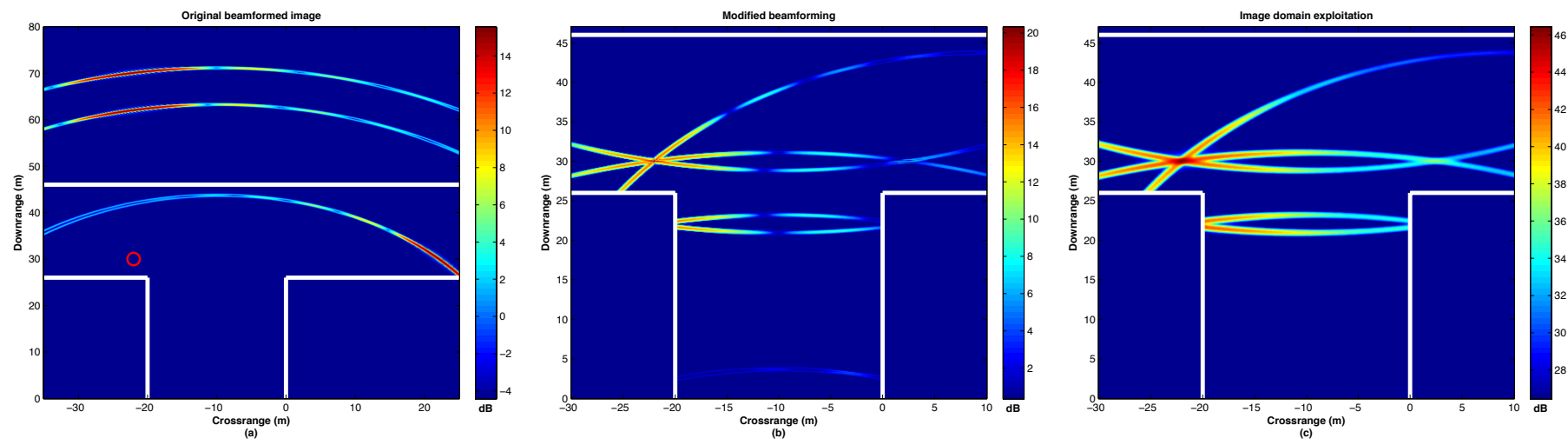

Fig. 5: For T-junction: (a) original beamformed, and multipath exploited (b) in data domain, (c) in image domain

in amplitude and seen in Fig. 7(a,b). The results for the image domain algorithm are not shown since it is robust to incoherent multipath at all locations, irrespective of the imaged geometry.

\section{PSF \& CPSF analysis:}

Fig. 8 depicts the CPSF, the CPSF interference interior to the imaged geometry, and the sidelobe of the PSF for an unshadowed target at $(-17,8)$ and $(-23,32)$, for the canyon and the T-junction respectively. Since the PSF has only the target mainlobe and the sidelobe, the aim here is to compare the CPSF interference w.r.t. to the PSF sidelobe.
The PSF sidelobe is derived numerically by first normalizing the image to its maximum, and then retaining only those contributions which are $6 \mathrm{~dB}$ lower than its maximum. We may see from Fig. 8 that the CPSF interference is generally spatially spread and is reasonable when compared to their corresponding PSF sidelobe. To quantify the CPSF interference we consider two metrics: the image variance and entropy of the CPSF interference images interior to the considered geometries [26],[27]. The metrics are compared to their PSF sidelobe counterparts in the image domain. The image variance measures the dispersion from the mean intensity in the image. 


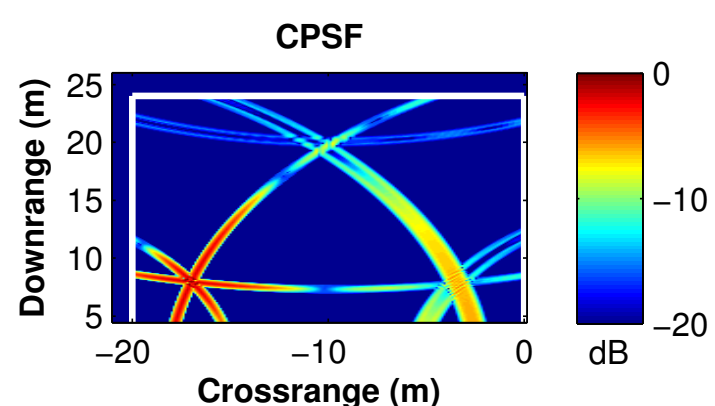

(a)

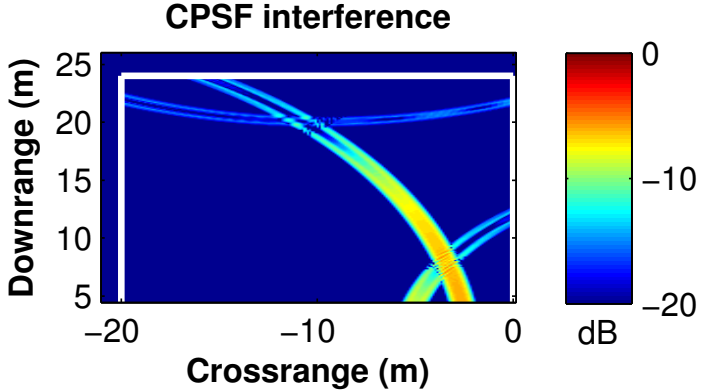

(b)

PSF sidelobe

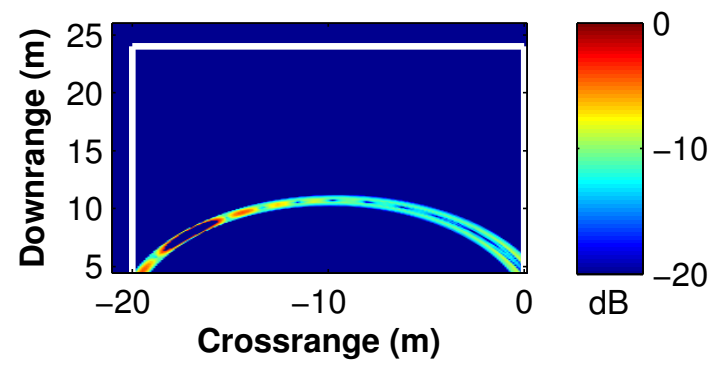

(c)

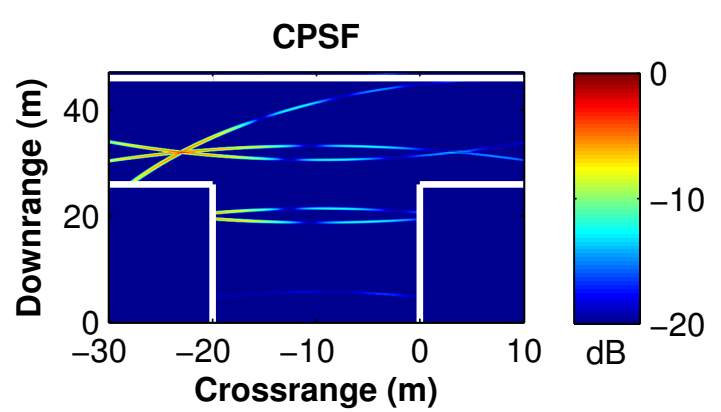

(d)

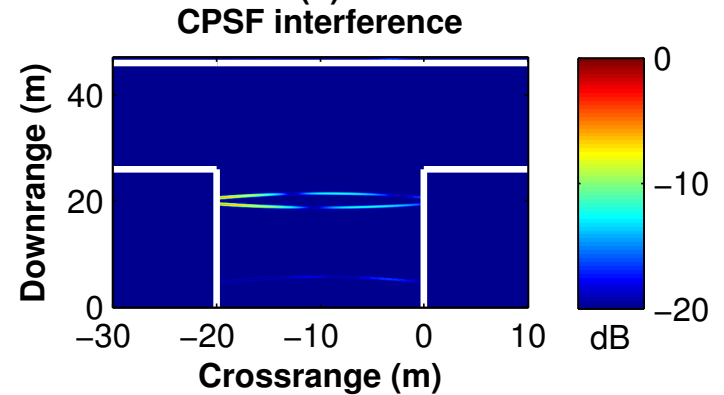

(e)

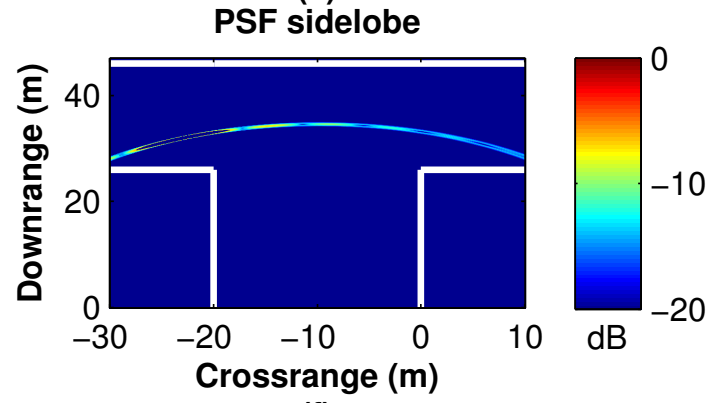

(f)

Fig. 8: Urban canyon target at $(-17,17)$ : (a) CPSF, (b) CPSF interference (c) PSF sidelobe, and T-junction target at $(-23,32)$ : (d) CPSF, (e) CPSF interference, (f) PSF sidelobe

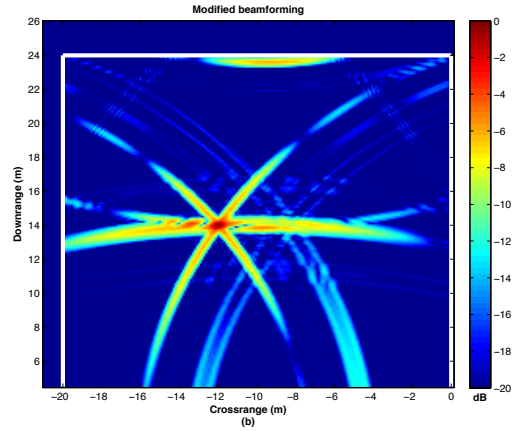

Fig. 6: Beamformed image after multipath exploitation with four multi path. The fourth multipath: propagation is via wall-1, return is via wall-3.

The entropy measures the randomness in intensities of the image.

Referring to Table IV, the variance of the CPSF interference is about the same as that of the PSF sidelobe for both geometries. The entropy is slightly higher for the urban canyon compared to the T-junction, which is explained from Fig. 8
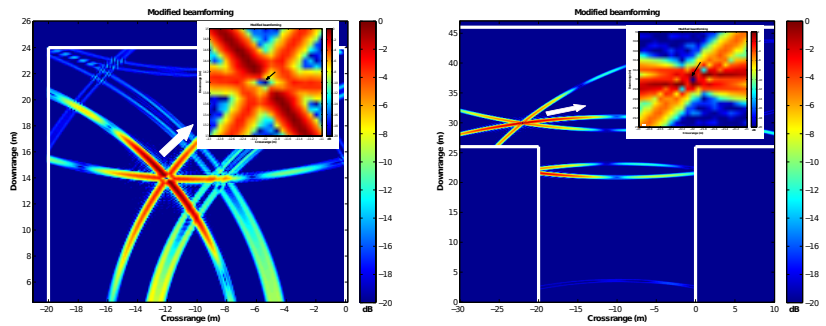

Fig. 7: Incoherent multipath scenario after multipath exploitation for (a) Urban canyon, (b) T-junction.

observing that the interference in the canyon is more spatially spread than the CPSF interference in the T-junction.

\section{E. Effects of noise and clutter, with undetectable multipath:}

We now consider adding independent white zero mean Gaussian complex noise to the radar returns resulting in an SNR of $3 \mathrm{~dB}$ at all sensors. Urban clutter is difficult to model statistically and physically, hence we assume sev- 
TABLE IV: PSF sidelobe and CPSF interference comparisons for target in urban canyon at $(-17,8)$ and in T-junction at $(-23,32)$.

\begin{tabular}{l|l||l||l}
\hline & & Urban Canyon & T-junction \\
\hline \hline \multirow{2}{*}{ Variance } & PSF sidelobe & 0.0267 & 0.0116 \\
\cline { 2 - 4 } & CPSF interference & 0.0354 & 0.0115 \\
\hline \multirow{2}{*}{$\begin{array}{l}\text { Entropy } \\
\text { (bits) }\end{array}$} & PSF sidelobe & 0.5334 & 0.2690 \\
\cline { 2 - 4 } & CPSF interference & 2.1176 & 0.499 \\
\hline \hline
\end{tabular}

eral scintillating and unshadowed point targets whose RCS varies randomly over the sensor positions. Note that the clutter targets assume a LOS return unlike the genuine target. The SCR was chosen to be $10 \mathrm{~dB}$. For the urban canyon, three clutter point targets were chosen at locations $(-18,22),(-18.5,22.5)$, and $(-19,23)$; their RCS are independent from each other, spatially independent across the sensors, and normally distributed. For the T-junction the clutter consisted of the same three scintillating point targets but at locations $(0,44),(-0.5,44.5)$, and $(-1,45)$. The locations of the clutter was assumed such that two of their multipath are not seen by the radar since they are at grazing angle, whereas their third multipath is assumed to be weak and below the noise floor, attributed to a low RCS of the target at this particular aspect angle. The aim here is twofold, to first demonstrate, unlike in [14], that clutter present inside the geometries are not mapped back inside by the two multipath exploitation algorithms. Second, to analyze the performance of the exploitation techniques in noise and clutter. Note that if interior clutter has its own multipath, then it is treated as a target and its multipath are mapped back by our exploitation techniques to the clutter location.

A simple cell averaging constant false alarm rate (CFAR) processor was used [28] in the image domain. After CFAR detection, dilation followed by erosion were carried out on the binary images [29],[30], to first retain clusters which should have been connected, and then locally erode clusters which are spatially far away [31]. These operations after raw CFAR detection are common and have been used in practical implementations in SAR CFAR detectors, see also [31],[32] and references therein for more details. The CFAR kernel is shown in Fig. 9(a), the structuring elements [30] for dilation and erosion are shown in Fig. 9(b,c). The test cell in Fig. 9(a) is square and has approximately the same length and width as the range resolution. The guard cells as shown in Fig. 9(a) are chosen hoping that no sidelobes contaminate the statistics in the test cell. The area comprising the guard and test cells is $2.64 \mathrm{~m}^{2}$. The training cells from which the statistics are derived span an area of $2.65 \mathrm{~m}^{2}$.

For the two geometries, the beamformed image before detection, and after detection at $2.5 \%$ and $1 \%$ false alarm rates are shown in Fig. 10(a,d,g) and Fig. 11(a,d,g), respectively. Some of the multipath and the clutter is seen clearly in Fig. 10(a) and Fig. 11(a), several false targets due to noise also appear, and are picked up by the CFAR as false targets in Fig. 10(d,g) and Fig. 11(d,g). For the urban canyon, the multipath w.r.t. wall 2 is not seen as it is intentionally made weak and below the noise floor, the rest of the multipath

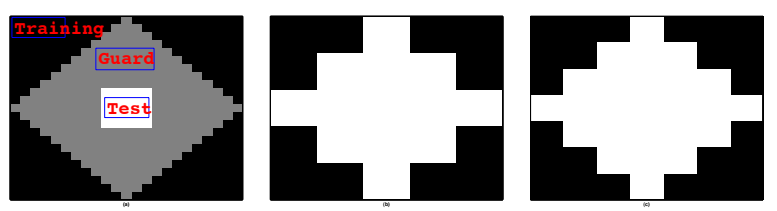

Fig. 9: CFAR operations, (a) kernel, (b) dilation structuring element, (c) erosion structuring element

are seen in Fig. 10(a). They are also seen in the data and image domain multipath exploitation algorithms in Fig. 10(e,f) and Fig. 10(h,i), and hence aid in localizing the target. Likewise for the T-junction, the farthest multipath is made weak. The remaining multipath and the clutter are seen in Fig. 11(a), and in the images after exploitation and detection i.e. in Fig. 11(e,f,h,i). The images in these figures are shown without normalization to depict the effect of both noise and clutter clearly. For the T-junction the detections are shown interior to this geometry following previous convention. From these figures it is clear that both the multipath exploitation algorithms localize and detect the shadowed target at its true location and that they lead to fewer false alarms inside the imaged geometries. It is noted that the target is detected in both the geometries, regardless of the false alarm rate.

The persistent clutter inside the canyon is seen in Fig. 10(a) but is not mapped back by the exploitation algorithms, regardless of the false alarm rate. The strong sidelobe from the clutter however is, and a small fraction of it is retained by the CFAR, especially by the data domain algorithm, but still goes undetected at the lower false alarm. For the T-junction, some of the clutter is mapped back since it is similar to the multipath from wall 2, which lies inside the T-junction. The clutter locations for the T-junction were therefore chosen to demonstrate this behavior intentionally. It is noted however that this clutter is mapped to locations which are well within the direct LOS view of the radar, and hence may be easily discounted from the CFAR detections. It must be noted that the CFAR uses local statistics for adapting its threshold and hence at the edges of the images, it performs poorly as seen here, as well as pointed out in the literature [31].

\section{F. Wall roughness:}

The operating frequency is now changed to $10.8 \mathrm{GHz}$. The standard deviations of the random variables modelling the roughness are chosen to be $10 \%$ of the chosen wavelength. The parameter $\kappa=0.04$ and number of craters in the walls is assumed to be $N=200$. The results are shown for varying values of $\varepsilon$ in Fig. 12(a-c) for the T-junction and for the data domain algorithm. The results for the image domain algorithm are similar and are therefore not shown. For the T-junction and with regard to the farthest multipath which has two reflections one at wall 3 and the other at wall 2, the diffuse multipath is modeled such that for a reflection at a particular crater in wall 3 , the signal may reach any other crater on wall 2 , reflect from it and then reach the target. From Fig. 12(a-c) we see that the wall roughness induces diffuse multipath longer in range than the specular component. From Fig. 12(a-c) we also 

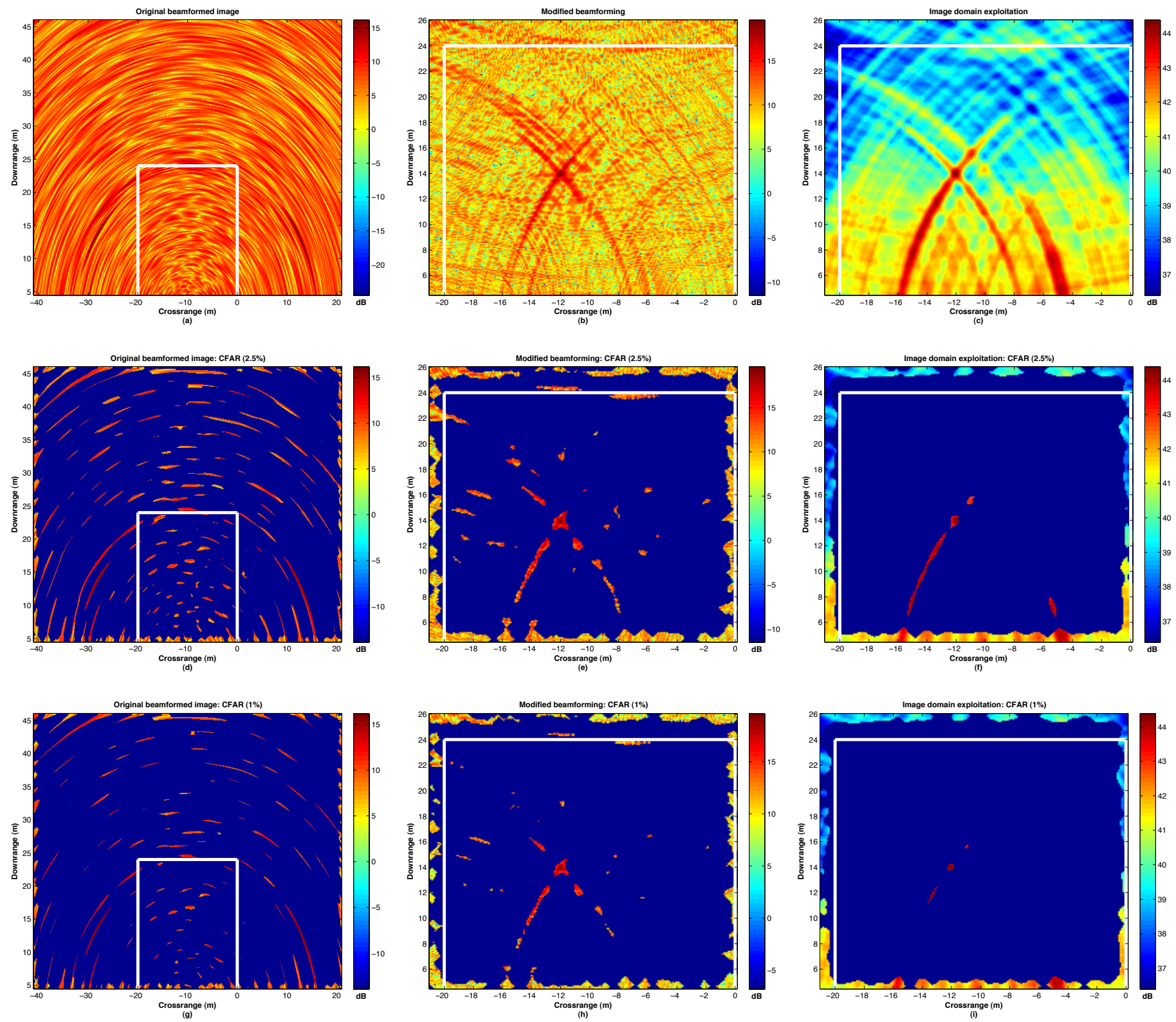

Fig. 10: Urban canyon CFAR results, for beamformed (a) original, (d) at $2.5 \%$,(g) at $1 \%$, for data domain (b) original,(e) at $2.5 \%,(\mathrm{~h})$ at $1 \%$, for image domain (c) original, (f) at $2.5 \%,(\mathrm{i})$ at $1 \%$

see that the diffuse multipath reduce with increasing values of $\varepsilon$. The range of acceptable values of $\varepsilon$ is an open question, which has to be addressed via experimental investigations or EM driven simulations; it is envisioned that the range of $\varepsilon$ would be a function of the material properties of the walls.

\section{G. Multiple closely resolvable targets:}

We next evaluate the performance when trying to resolve two closely spaced shadowed targets. For the urban canyon the targets are at $(-12,14)$ and $(-12.7,14.7)$ and for the T-junction the targets are at $(-22,30)$ and $(-22.7,30.7)$. For such closely spaced targets, some of their corresponding multipath are also unresolvable. For the urban canyon in Fig. 13(a), we see that the multipath w.r.t. wall 3 is unresolved for both the targets. The unresolved multipath is remapped as in Fig. 13(b,c). From the data and image domain exploited images, we see that the target has been resolved. For the T-junction, in Fig. 14(a), the furthest multipath are more or less unresolved. The data domain exploited image does not reveal two targets but rather four closely separated peaks, due to several criss-crossed multipath trajectories. Two of these are at the true location, whereas the others manifest due to intersections of the original multipath trajectories. The image domain exploited technique nevertheless shows two sharp peaks at the true target locations. This is attributed to the spatial integration utilizing the PSF. 

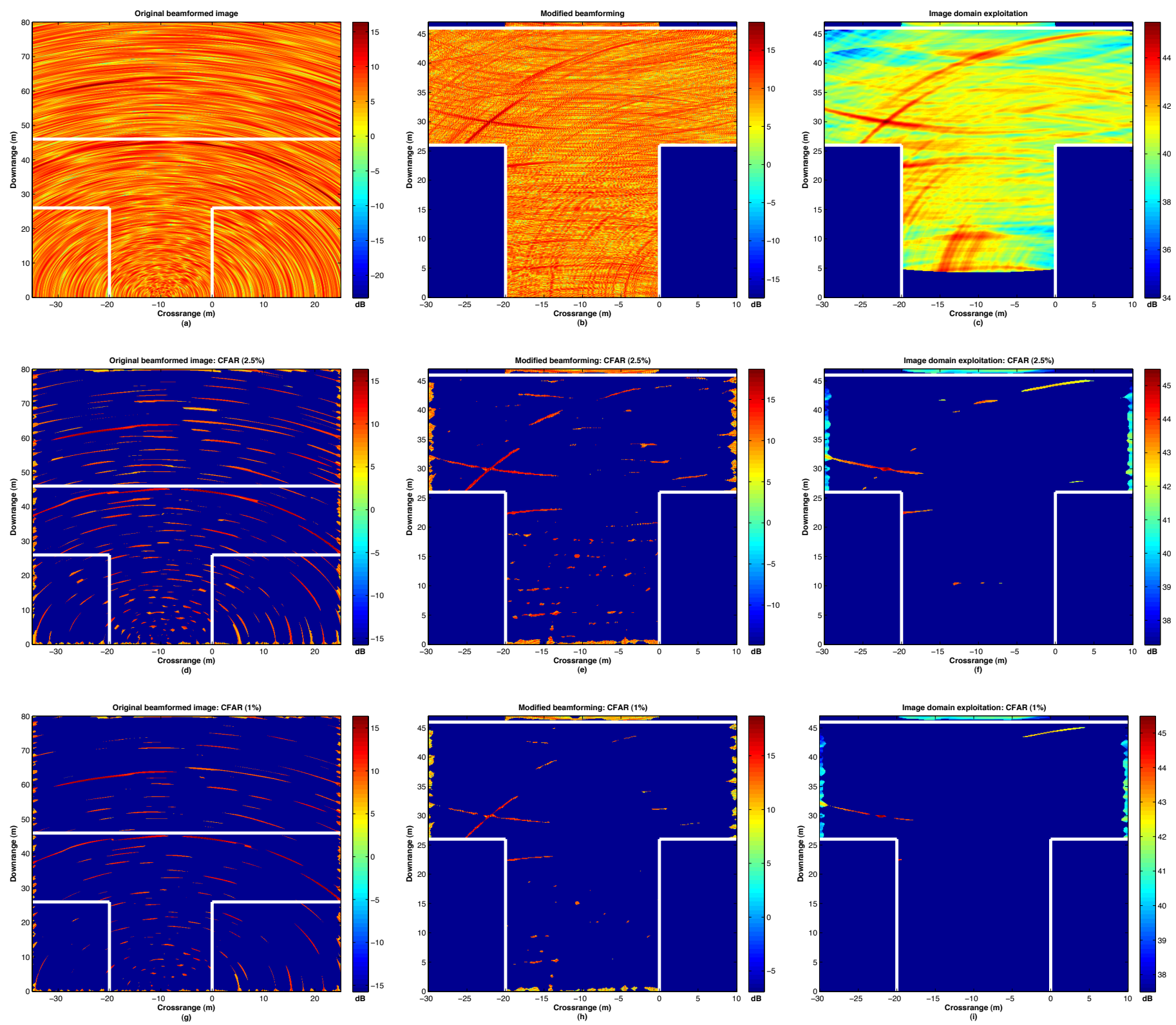

Fig. 11: T-junction CFAR results, for beamformed (a) original, (d) at $2.5 \%$, (g) at $1 \%$, for data domain (b) original, (e)at $2.5 \%$,(h) at $1 \%$, for image domain (c) original, (f) at $2.5 \%$, (i) at $1 \%$
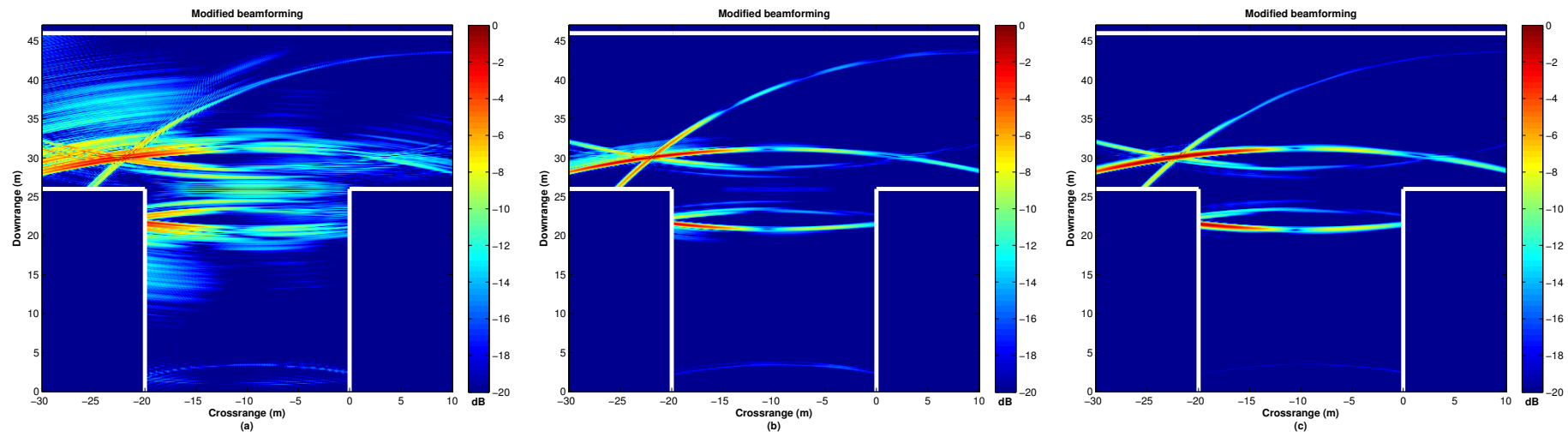

Fig. 12: Rough wall results for T-junction and data domain algorithm, (a) $\varepsilon=0.05$, (b) $\varepsilon=0.1$, (c) $\varepsilon=0.15$ 

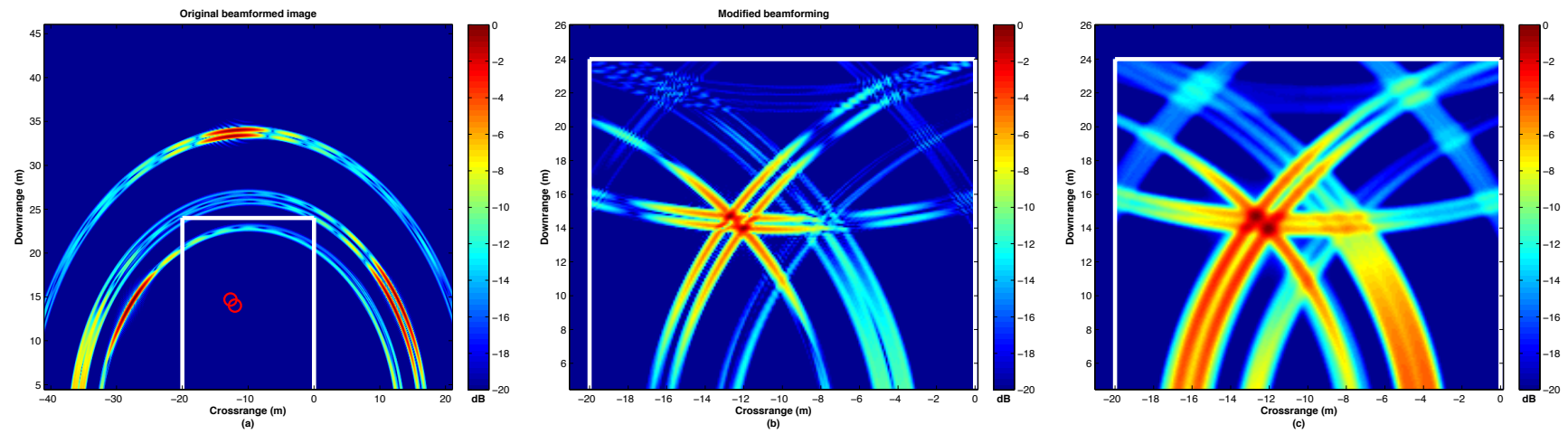

Fig. 13: For Urban canyon two closely separated targets: (a) original beamformed, and after multipath exploitation (b) data domain, (c) image domain
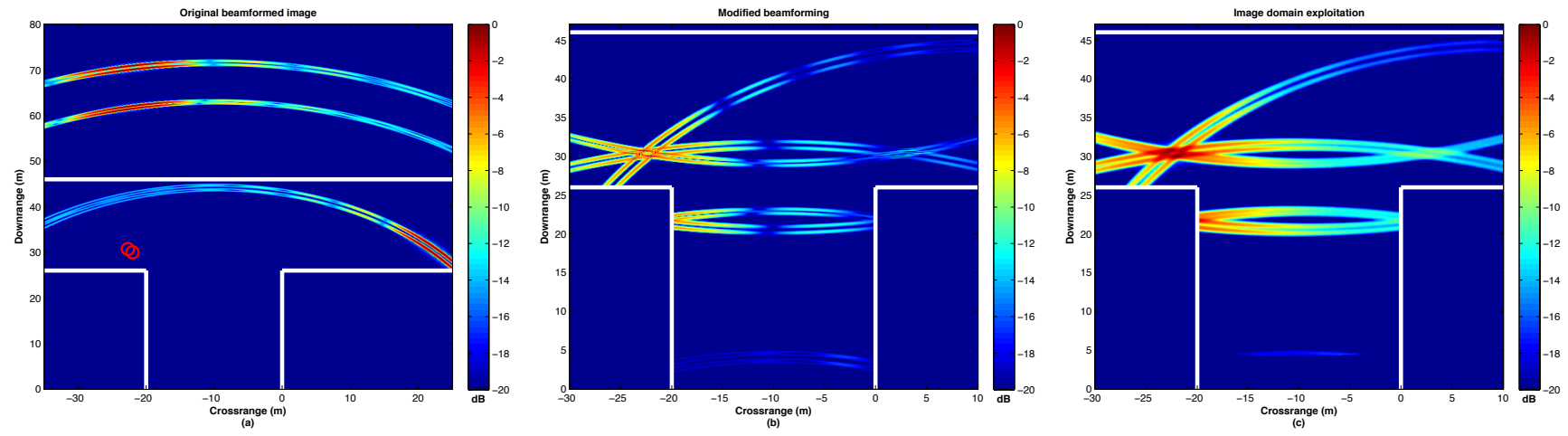

Fig. 14: For T-junction, two closely separated targets: (a) original beamformed, and after multipath exploitation (b) data domain, (c) image domain

\section{H. Inaccuracies in wall positions}

For the urban canyon, we assume that wall 1 and wall 3 are off by $+0.5 \mathrm{~m}$ in their locations, and similarly wall 2 is $-0.5 \mathrm{~m}$ in error. For the T-junction we assume that the wall to the right of the radar has an error of $+0.5 \mathrm{~m}$ in its location. Likewise, we assume that $D_{1}$ and $D_{3}$ have errors corresponding to $\pm 0.5 \mathrm{~m}$ respectively. The results are shown for the data domain multipath exploitation in Fig. 15 along with the true target location. The errors in wall positions for both the urban canyon and T-junction, corresponds to approximately seventeen wavelengths for a carrier of $10 \mathrm{GHz}$, and is reasonably large. For these simulations we observe that when the wall positions are in error, the exploited multipath do not all intersect at the true target location. It is also noted from this figure that due to the nonoverlapping trajectories arising from the errors in wall positions, there is not a significant gain in SNR at the true target location when compared to the case of exploiting multipath when no errors in wall positions exist. This implies that some benefits by exploiting multipath could be lost especially in lower SCR / SNR regimes.

Simulations with the CPSF (without the spatial interference) in the presence of geometry errors are shown in Fig. 16 and in Fig. 17, for the two urban structures. For these simulations $\alpha=3 \mathrm{~dB}$ was chosen (see Appendix-B). When at least some of the multipath intersect (the (b) sub-figures), the norm squared error in the target location is $2.34 \mathrm{~m}^{2}$ and $16.6 \mathrm{~m}^{2}$, for the urban canyon and T-junction respectively. When none of the multipath intersect (the (a) sub-figures), the uncertainty polygons, $\mathcal{D}$ are shown in these figures.

\section{CONCLUSions}

The objective of this article was to localize and detect targets in the presence of multipath but in the absence of the direct LOS path, assuming that knowledge about the reflecting walls is available. Two multipath exploitation techniques were formulated, the first operates on the original beamformed image, whereas the other operated on the raw data in the signal domain, and both relied on multipath being detectable. It was shown that traditional processing of multipath causes false alarms in the beamformed image. However, after our proposed multipath exploitation, the target was detected and localized at its true location, and the false alarms were reduced while simultaneously increasing the SCR at the target location.

The impact of radar multipath from rough walls was modeled stochastically and its impact was shown via representative simulations. Effectively exploiting multipath for localizing and detecting targets, currently requires fairly accurate estimates of the geometry and blueprints of the urban structures. To addresses inaccuracies in geometries, analysis and simulations were considered. The simulations demonstrate that for 
reasonable measurement errors multipath exploitation still holds some promise. However, the robustness of multipath exploitation schemes in the absence of direct LOS path to diffuse multipath from other objects and diffraction effects were not addressed analytically, and additional investigation and experimental work would be highly desired to address their detrimental effects on multipath exploitation.

\section{APPENDIX A}

CPSF AND PSF FOR RECTANGULAR WAVEFORM

Let us assume that $p(t)$ is rectangular function of width $T$,

$$
\begin{gathered}
p(t)=\operatorname{rect}(t / T):= \begin{cases}1 & 0 \leq t \leq T \\
0 & \text { otherwise }\end{cases} \\
P(\omega)=T \exp (-j \omega T / 2) \operatorname{Sinc}(\omega T / 2 \pi)
\end{gathered}
$$

Then the PSF for the rectangular function, $\mathcal{P}^{r}\left(\mathbf{x}_{t}, \mathbf{x}\right)$, is:

$$
\mathcal{P}^{r}\left(\mathbf{x}_{t}, \mathbf{x}\right)=\sum_{m=1}^{M} \Psi\left(\Delta \tau_{m}\left(\mathbf{x}_{t} ; \mathbf{x}\right)\right)
$$

where

$$
\begin{aligned}
& \Psi\left(\Delta \tau_{m}\left(\mathbf{x}_{t} ; \mathbf{x}\right)\right):= \begin{cases}\Phi_{1}(\cdot) & \frac{\left|\Delta R_{m}\left(\mathbf{x}_{t} ; \mathbf{x}\right)\right|}{\Delta R} \leq 1 \\
0 & \text { otherwise }\end{cases} \\
& \Delta R_{m}\left(\mathbf{x}_{t} ; \mathbf{x}\right)=c \Delta \tau_{m}\left(\mathbf{x}_{t} ; \mathbf{x}\right), \Delta R=\frac{c T}{2}=\frac{c}{2 B} \\
& \Phi_{1}(\cdot)=T\left(1-\frac{\left|\Delta R_{m}\left(\mathbf{x}_{t} ; \mathbf{x}\right)\right|}{\Delta R}\right) \exp \left(-j \omega_{c} 2 \Delta \tau_{m}\left(\mathbf{x}_{t} ; \mathbf{x}\right)\right)
\end{aligned}
$$

and $\Delta R$ is the range resolution of the system for the radar bandwidth denoted by parameter $B$. The PSF captures the range resolution via $\Psi\left(\Delta \tau_{m}\left(\mathbf{x}_{t} ; \mathbf{x}\right)\right)$ and takes into account oversampling in downrange and crossrange, if they were employed. With similar convention, the CPSF specialized for the rectangular function is

$$
\mathcal{P}_{c}^{r}\left(\mathbf{x}_{t}, \mathbf{x}\right)=\sum_{m=1}^{M} \sum_{k=1}^{3} \sum_{l=1}^{3} \Psi\left(\Delta \tau_{m k l}\left(\chi_{k}^{v t} ; \overline{\boldsymbol{\chi}}_{l}^{v t}\right)\right)
$$

where $\Psi\left(\Delta \tau_{m k l}\left(\chi_{k}^{v t} ; \bar{\chi}_{l}^{v t}\right)\right)$ is only defined for the multipath and is given by

$\Psi\left(\Delta \tau_{m k l}\left(\chi_{k}^{v t} ; \overline{\boldsymbol{\chi}}_{l}^{v t}\right)\right):= \begin{cases}\Phi_{2}(\cdot) & \frac{\left|\Delta R_{m k l}\left(\chi_{k}^{v t} ; \overline{\boldsymbol{\chi}}_{l}^{v t}\right)\right|}{\Delta R} \leq 1 \\ 0 & \text { otherwise }\end{cases}$

$\Delta R_{m k l}\left(\chi_{k}^{v t} ; \bar{\chi}_{l}^{v t}\right)=c \tau_{m k l}\left(\chi_{k}^{v t} ; \bar{\chi}_{l}^{v t}\right)$

$\Phi_{2}(\cdot)=T\left(1-\frac{\left|\Delta R_{m k l}\left(\boldsymbol{\chi}_{k}^{v t} ; \overline{\boldsymbol{\chi}}_{l}^{v t}\right)\right|}{\Delta R}\right) \exp \left(-j \omega_{c} 2 \Delta \tau_{m k l}\left(\boldsymbol{\chi}_{k}^{v t} ; \overline{\boldsymbol{\chi}}_{l}^{v t}\right)\right.$

We have all the necessary terms to compute the interference term in the CPSF specialized for the rectangular function, and is given by

$$
\begin{aligned}
& \mathcal{P}_{c}\left(\mathbf{x}_{t}, \mathbf{x}\right)-\mathcal{Q}_{c}\left(\mathbf{x}_{t}, \mathbf{x}\right) \\
& =\sum_{m=1}^{M} \sum_{k=1}^{3} \sum_{l=1}^{3} \Psi\left(\Delta \tau_{m k l}\left(\chi_{k}^{v t} ; \overline{\boldsymbol{\chi}}_{l}^{v t}\right)\right) \\
& -\sum_{m=1}^{M} \sum_{k=1}^{3} \Psi\left(\Delta \tau_{m k k}\left(\boldsymbol{\chi}_{k}^{v t} ; \overline{\boldsymbol{\chi}}_{k}^{v t}\right)\right)
\end{aligned}
$$

Numerical results of the rectangular PSF and CPSF are presented in Section IV. In particular, it is shown that in certain cases the interference term is spatially spread in both downrange and crossrange, and its magnitude is more or less insignificant when compared to the sidelobe levels of the original PSF.

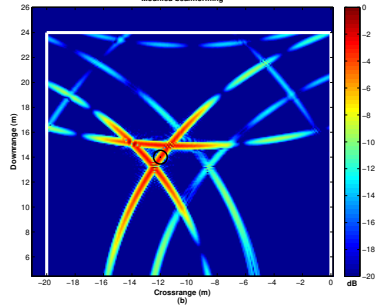

(a)

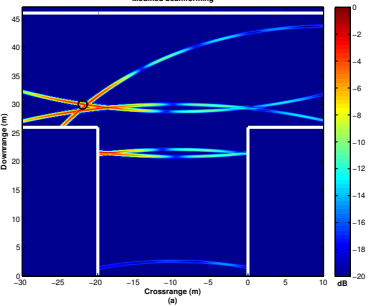

(b)
Fig. 15: Multipath exploitation for the data domain algorithm, when wall positions are in error, (a) Urban canyon, (b) T-Junction. The true target position is shown by $(\bigcirc)$

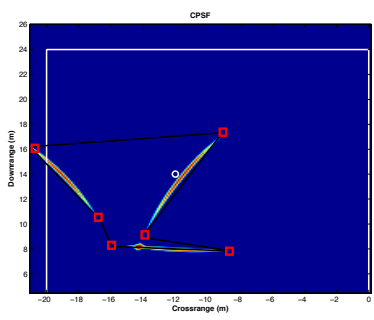

(a)

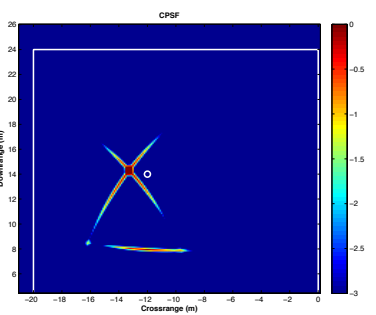

(b)
Fig. 16: CPSF without the spatial interference in the presence of geometry errors for the urban canyon. The true target position is at $(\bigcirc)$ : (a) $\Delta_{1}=0.5, \Delta_{2}=-3, \Delta_{3}=3.5$, the uncertainty polygon, $\mathcal{D}$ in black and its vertices $(\square)$ are also shown. (b) $\Delta_{1}=-1.5, \Delta_{2}=-3, \Delta_{3}=0.5$

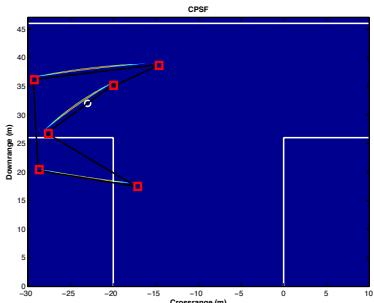

(a)

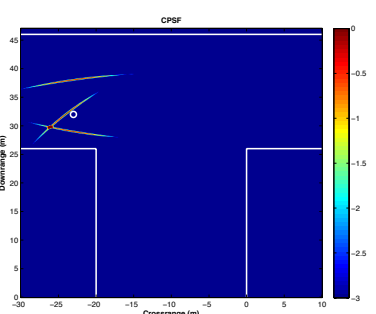

(b)
Fig. 17: CPSF without the spatial interference in the presence of geometry errors for the T-junction. The true target position is at $(\bigcirc):$ (a) $\Delta_{1}=-1, \Delta_{2}=-3.5, \Delta_{3}=-3$, the uncertainty polygon, $\mathcal{D}$ in black and its vertices $(\square)$ are also shown. (b) $\Delta_{1}=-1, \Delta_{2}=1.5, \Delta_{3}=-3$ 


\section{APPENDIX B}

\section{SENSITIVITY ANALYSIS: ERRORS IN GEOMETRY}

The objective is to provide a mathematical framework to analyze the impact of imprecise knowledge of the geometry (or geometry errors) on the presented multipath exploitation approach. We consider a single target and $K$ distinguishable multipath components. We assume that the wall position errors, denoted as $\Delta_{i}, i=1,2 \ldots$, will result in virtual target position errors for each of the $K$ multi path components.

Depending on the exact types of errors, either some of the $K$ multipath trajectories will still intersect in the modified beam formed image, or, in the worst case, none of them will intersect in the modified image. To analyze both these cases, the CPSF without the spatial interference proves to be useful. Consider (13), looking at each multi path component $k=1,2, \ldots, K$ multipath individually, define:

$$
\begin{aligned}
& \mathcal{Q}_{c k}\left(\mathbf{x}_{t}, \mathbf{x}\right)=\sum_{m=1}^{M} \mathcal{F}^{-1}\left\{\left|P\left(\omega-\omega_{c}\right)\right|^{2}\right. \\
& \times \exp \left(-j\left(\omega-\omega_{c}\right) 2 \Delta \tau_{m k k}\left(\boldsymbol{\chi}_{k}^{v t} ; \dot{\bar{\chi}}_{k}^{v t}\right)\right) \\
& \left.\times \exp \left(-j \omega_{c} 2 \Delta \tau_{m k k}\left(\chi_{k}^{v t} ; \dot{\bar{\chi}}_{k}^{v t}\right)\right)\right\}\left.\right|_{t=0} .
\end{aligned}
$$

In (24), $\chi_{k}^{v t}$ is the $k$-th virtual target in the absence of errors for the target at the true location at $\mathrm{x}_{t}$, and $\dot{\bar{\chi}}_{k}^{v t}$ is the $k$-th virtual target for a hypothesized target at $\mathbf{x}$ but in the presence of geometry errors. The rest of the parameters in (24) have been previously defined. For the specific case of the urban canyon, and for $K=3$, we have $\dot{\bar{\chi}}_{1}^{v t}=\left[x_{t}+\Delta_{1}, y_{t}\right]^{T}, \dot{\bar{\chi}}_{2}^{v t}=$ $\left.\left[-x_{t}, 2\left(D_{1}+\Delta_{2}+D_{y}\right)-y_{t}\right)\right]^{T}$ and $\dot{\bar{\chi}}_{3}^{v t}=\left[-\left(2\left(D_{2}+\Delta_{3}\right)-\right.\right.$ $\left.\left.x_{t}\right), y_{t}\right]^{T}$. Similarly, for the T-junction and for $K=3$, we have $\dot{\bar{\chi}}_{1}^{v t}=\left[x_{t}+\Delta_{1}, y_{t}\right]^{T}, \dot{\bar{\chi}}_{2}^{v t}=\left[-x_{t}, 2\left(D_{3}+\Delta_{3}+D_{1}+\Delta_{2}\right)-\right.$ $\left.y_{t}\right]^{T}$, and $\dot{\bar{\chi}}_{3}^{v t}=\left[-x_{t}, 2\left(D_{3}+\Delta_{3}\right)+, y_{t}\right]^{T}$. For both these urban structures, the errors in wall positions are captured by $\Delta_{k}, k=1,2, \ldots$. Consider the following sets, corresponding to a chosen $\alpha$, for each multi path component $k$,

$$
\mathcal{A}_{k}:=\left\{\mathbf{x}=(x, y): \frac{\left|\mathcal{Q}_{c k}\left(\mathbf{x}_{t}, \mathbf{x}\right)\right|}{\max _{\mathbf{x}}\left|\mathcal{Q}_{c k}\left(\mathbf{x}_{t}, \mathbf{x}\right)\right|} \leq \alpha\right\}
$$

Typically, $\alpha \in[1 / 2,1 / 4]$ in linear scale or $\alpha \in[-3,-6] \mathrm{dB}$ is chosen to retain purely mainlobe multipath contributions.

When at least some of the multipath trajectories intersect, we need to consider at the very least, pairwise set intersections: $\mathcal{B}_{k j}:=\mathcal{A}_{k} \cap \mathcal{A}_{j}, j=k+1, k+2, \ldots, K$ with $k=1,2, \ldots, K$. There are a total of $\left(\begin{array}{c}K \\ 2\end{array}\right)$ such pairwise intersection sets. If at least some of the multipath trajectories intersect, then those intersection points could be considered as hypothesized target locations and are collected in the set $\mathcal{B}$, defined by:

$$
\mathcal{B}:=\bigcup_{k, j} \mathcal{B}_{k j}, k=1,2 \ldots, K, j=k+1, k+2, \ldots, K
$$

We can now define a metric such as the norm squared error between the true target location $\mathrm{x}_{t}$ and the members of $\mathcal{B}$ to determine the sensitivity of multipath exploitation to errors in geometry w.r.t localization. In the other extreme case, when none of the multipath trajectories intersect, $\mathcal{B}_{k j}=\{\emptyset\}$ for all $k, j$. In this case, we can declare the target to be anywhere in a region. It is natural to assume that, in this region, individual multipath mainlobes are connected at least partially to themselves, and furthermore individual multipath are connected to the rest of the multipath mainlobes either directly or via the other multipath mainlobes. The next step is to minimize the area (or volume, if $3 \mathrm{D}$ ) of this region ${ }^{1}$. From our extensive simulations, we noticed that a mathematically tractable shape to describe this region is an irregular polygon, with a minimum of $2 K$ sides to: a) at least partially connect individual multipath mainlobes, b) connect individual multipath to the rest either directly or via the other multipath.

Denote the vertices of $\mathcal{D}$ as $\hat{\mathbf{v}}_{k} \in \mathcal{A}_{k}$ and $\breve{\mathbf{v}}_{k} \in \mathcal{A}_{k}, k=$ $1,2, \ldots, K$, and are defined as:

$\begin{cases}\hat{\mathbf{v}}_{k}:=\left(x_{k 0}, y_{k 0}\right), \breve{\mathbf{v}}_{k}:=\left(x_{k 1}, y_{k 1}\right) & \text { if }\left|x_{k 0}-x_{k 1}\right| \geq\left|\bar{y}_{k 0}-\bar{y}_{k 1}\right| \\ \hat{\mathbf{v}}_{k}:=\left(\bar{x}_{k 0}, \bar{y}_{k 0}\right), \breve{\mathbf{v}}_{k}:=\left(\bar{x}_{k 1}, \bar{y}_{k 1}\right) & \text { if }\left|x_{k 0}-x_{k 1}\right|<\left|\bar{y}_{k 0}-\bar{y}_{k 1}\right|\end{cases}$

where $\mathcal{A}_{k}:=\left\{\left(x_{k}, y_{k}\right)\right\}$, and $\left(x_{k 0}, y_{k 0}\right)=\sup \left\{\left(x_{k}, y_{k}\right)\right\}$, $\left(x_{k 1}, y_{k 1}\right)=\inf _{x_{k}}\left\{\left(x_{k}, y_{k}\right)\right\}$. Similarly, $\quad \stackrel{x_{k}}{\left.\bar{x}_{k 0}, \bar{y}_{k 0}\right)=}$ $\sup _{y_{k}}\left\{\left(x_{k}, y_{k}\right)\right\},\left(\bar{x}_{k 1}, \bar{y}_{k 1}\right)=\inf _{y_{k}}\left\{\left(x_{k}, y_{k}\right)\right\}$. The above definition assumes that for example, $\left(x_{k 0}, y_{k 0}\right)=\sup _{x_{k}}\left\{\left(x_{k}, y_{k}\right)\right\}$ is unique, if this is not the case, any of the points could be used as the vertex. The important point is to ensure that exactly $K$ sides of $\mathcal{D}$ intersect the region/set $\mathcal{A}_{k}$ or are in close proximity to it. A few examples in Section V make this clear.

\section{ACKNOWLEDGMENT}

The authors wish to acknowledge Steven Scarborough, David Sobota and Muralidhar Rangaswamy from the US AFRL for their comments and discussions related to multipath exploitation in radar. The work of P. Setlur and N. Devroye was partially supported by AFOSR under award FA9550-101-0239. The work of T. Negishi and D. Erricolo was partially supported by AFOSR under award FA9550-12-1-0174.

\section{REFERENCES}

[1] A. Sume, M. Gustafsson, M. Herberthson, A. Janis, S. Nilsson, J. Rahm, and A. Orbom, "Radar Detection of Moving Targets Behind Corners," IEEE Trans. Geosci. and Rem. Sensing, vol. 49. no. 6, pp. 2259-2267 June 2011.

[2] D. Deiana, A. S. Kossen, and W. L. van Rossum,"Multipath exploitation in an urban environment using a MIMO surveillance radar," In Proc. Int. Radar Symp., 16-18 June. 2010.

[3] Z. Yun, S. Y. Lim, and M. F. Iskander, "Use of Geospatial Resources for Radio Propagation Prediction in Urban Areas," IEEE Antennas and Wireless Propagation Letters, Vol. 8, pp. 587-591, 2009.

[4] H. T. Hayvaci, A. De Maio, D. Erricolo, "Improved Detection Probability of a Radar Target in the Presence of Multipath with Prior Knowledge of the Environment," IET Radar, Sonar \& Navigation, Vol. 7 (1), 2013, pp. 36-46.

[5] B. Chakraborty, Y. Li, J. J. Zhang, T. Trueblood, A. PapandreouSuppappola, and D. Morrell, "Multipath exploitation with adaptive waveform design for tracking in urban terrain," In Proc. ICASSP, Dallas, TX, Mar. 2010.

[6] R. Linnehan, and J. Schindler, "Multistatic scattering from moving targets in multipath environments," In Proc. IEEE Inter. Radar Conf., Pasadena, California, 4-8 May 2009.

[7] S. Sen, M. Hurtado, and A. Nehorai, "Adaptive OFDM radar for detecting a moving target in urban scenarios," In Proc. Waveform Design and Diversity Conf., Kissimmee, Florida, 8-13 Feb. 2009.

${ }^{1}$ Disjoint regions minimizing the area could be derived but their construction is not easily described, and are dependent on the particular errors in the wall locations. Furthermore, the motivation to is to create a contiguous region since when we know all wall positions, the multipath all intersect. 
[8] M. M. Nikolic, A. Nehorai, and A. R. Djordjevic"Exploiting multipath from airborne platforms for direction of arrival estimation," In Proc. Eur. Conf. on Ant. and Prop., 23-27 Mar. 2009.

[9] J. L. Krolik, J. Farell, and A. Steinhardt, "Exploiting multipath propagation for GMTI in urban environments," In Proc. IEEE Radar Conf., Verona, NY, 24-27 Apr. 2006.

[10] D. A. Garren, J. S Goldstein, D. R. Obuchon, R. R Greene, and J. A. North, "SAR image formation with multipath reflectivity estimation," In Proc. Radar Conf., 26-29 Apr. 2004.

[11] D. A Garren and R. R Greene, "Method for developing and using an image reconstruction algorithm for multipath scattering," US patent, 7,259,715, filed, Oct 19, 2004, granted, Aug 21, 2007.

[12] T. Dogaru, and C. Le, "SAR images of rooms and buildings based on FDTD computer models," IEEE Trans. Geosci. and Remote Sensing, vol. 47, no. 5, pp. 1388-1401, 2008.

[13] S. Kidera, T. Sakamoto, and T. Sato, "Experimental study of shadow region imaging algorithm with multiple scattered waves for UWB radars," PIERS Online, vol. 5, no. 4, pp. 393-296, 2009.

[14] P. Setlur, M. Amin, F. Ahmad, "Multipath model and exploitation in through-the-wall and urban radar sensing," IEEE Trans. Geosci. and Rem. Sensing, vol. 49, no. 10, pp. 4021-4034, Oct. 2011.

[15] P. Setlur, G. Alli and L. Nuzzo, "Multipath Exploitation in ThroughWall Radar Imaging Via Point Spread Functions," IEEE Trans. Image Proc., In Press, 2013.

[16] M. I. Skolnik, Radar Handbook, Second Edition, McGraw Hill, New York, NY, 1990.

[17] M. Soumekh, Synthetic Aperture Radar Signal Processing with MATLAB Algorithms, John Wiley \& Sons, Inc., New York, NY, 1999.

[18] A.F. Yegulalp, "Fast backprojection algorithm for synthetic aperture radar," In Proc. IEEE Radar Conf., pg. 60-65, 1999.

[19] M. D. Desai and W. K. Jenkins, "Convolution backprojection image reconstruction for spotlight mode synthetic aperture radar," IEEE Trans. Image Proc., vol. 1, no. 4, pp. 505-517, Oct. 1992.

[20] L. M. H. Ulander, H. Hellsten, and G. Stenstrom, "Synthetic-aperture radar processing using fast factorized back projection," IEEE Trans. Aerosp. Electron. Syst., vol. 39 no. 3, pp. 760-776, July 2003.

[21] G. E. Smith, B. G. Mobasseri, "Robust Through-the-Wall Radar Image Classification Using a Target-Model Alignment Procedure," IEEE Trans. Image Proc., vol. 21, no. 2, pp. 754-767, Feb. 2012.

[22] S. O. Rice, "Reflection of Electromagnetic waves from slightly rough surfaces," Comm. Pure Appl. Math, vol. 4, no. 2/3, pp. 351-378, 1951.

[23] W. S. Ament, "Forward and back-scattering from certain rough surfaces," IEEE Trans. Ant. and Prop., vol. 4, no. 3, pp. 369-373, Jul. 1956.

[24] A. Tabatabaeenejad and M. Moghaddam,"Bistatic scattering from threedimensional layered rough surfaces," IEEE Trans. GeoSci. Rem. Sen., vol. 44, no. 8, pp. 2102-2114, Aug. 2006.

[25] A. K. Jain, "Advances in Mathematical Models for Image Processing," Proc. of the IEEE, vol. 69, no. 5, pp. 502-528, May 1981.

[26] C. Oliver and S. Quegan, Understanding Synthetic Aperture Radar Images, SciTech Publishing, Raleigh, NC, Jan. 2004.

[27] I. Avcbaş, B. Sankur, and K. Sayood,"Statistical evaluation of image quality measures," Journ. Electron. Imaging, vol. 11, no. 2, pp. 206223, Apr. 2002.

[28] H. Rohling, "Radar CFAR thresholding in clutter and multiple target situations," IEEE Trans. Aerosp. and Electron. Syst., vol. 19. no. 4, pp. 608-621, Jul. 1983.

[29] A. K. Jain, Fundamentals of Digital Image Processing, Prentice Hall, 1988.

[30] E. R. Dougherty and R. A. Lotufo, Hands-on Morphological Image Processing SPIE, Bellingham, WA, 2003.

[31] A. Martone, K. Ranney, and R. Innocenti, "Automatic through the wall detection of moving targets using low frequency ultra wideband radar," In Proc. IEEE Radar Conf. Washington D.C, May 10-14, 2010.

[32] D. J. Crisp, "The state-of-the-art in ship detection in synthetic aperture radar imagery," Defence Sci. Technol. Org., Port Wakefield, South Australia, Research Report DSTO-RR-0272, May 2004. Available at: http://www.dsto.defence.gov.au/corporate/reports 